\title{
Far-infrared emission from intracluster dust in Abell clusters ${ }^{\star}$
}

\author{
M. Stickel ${ }^{1}$, U. Klaas ${ }^{1}$, D. Lemke ${ }^{1}$, and K. Mattila ${ }^{2}$ \\ 1 Max-Planck-Institut für Astronomie, Königstuhl 17, 69117 Heidelberg, Germany \\ 2 Helsinki University Observatory, PO Box 14, 00014 Helsinki, Finland
}

Received 27 July 2001 / Accepted 7 November 2001

\begin{abstract}
The ISOPHOT instrument aboard ISO has been used to observe extended FIR emission of six Abell clusters. Strip scanning measurements with crossing position angles centered on the clusters were carried out at $120 \mu \mathrm{m}$ and $180 \mu \mathrm{m}$. The raw profiles of the $I_{120 \mu \mathrm{m}} / I_{180 \mu \mathrm{m}}$ surface brightness ratio including zodiacal light show a bump towards Abell 1656 (Coma), dips towards Abell 262 and Abell 2670, and are without clear structure towards Abell 400, Abell 496, and Abell 4038. After subtraction of the zodiacal light, the bump towards Abell 1656 is still present, while the dips towards Abell 262 and Abell 2670 are no longer noticable. This indicates a localized excess of emitting material outside the Galaxy towards Abell 1656 with properties different from the galactic foreground cirrus, while the behavior in Abell 262 and Abell 2670 can be reconciled with galactic cirrus structures localized on the line-of-sight to these clusters. The excess of $\approx 0.2 \mathrm{MJy} / \mathrm{sr}$ seen at $120 \mu \mathrm{m}$ towards Abell 1656 (Coma) is interpreted as being due to thermal emission from intracluster dust distributed in the hot X-ray emitting intracluster medium. The integrated excess flux within the central region of $10^{\prime}-15^{\prime}$ diameter is $\approx 2.8 \mathrm{Jy}$. Since the dust temperature is poorly constrained, only a rough estimate of the associated dust mass of $M_{\mathrm{D}} \approx 10^{7} M_{\odot}$ can be derived. The associated visual extinction is negligible $\left(A_{V} \ll 0.1 \mathrm{mag}\right)$ and much smaller than claimed from optical observations. No evidence is found for intracluster dust in the other five clusters observed. The absence of any signature for intracluster dust in five clusters and the rather low inferred dust mass in Abell 1656 indicates that intracluster dust is likely not responsible for the excess X-ray absorption seen in cooling flow clusters. These observations thereby represent a further unsuccessful attempt in detecting the presumed final stage of the cooling flow material, in accord with quite a number of previous studies in other wavelengths regions. Finally, the observed dimming of the high-redshift supernovae is unlikely be attributable to extinction caused by dust in the intracluster or even a presumed intercluster medium.
\end{abstract}

Key words. galaxies: clusters: general - galaxies: clusters: individual: Abell 262, Abell 1656, Abell 2670 intergalactic medium - infrared: general

\section{Introduction}

The analysis of the X-ray and UV emission of the hot $\left(T_{\mathrm{e}} \approx 1-10 \mathrm{keV}\right)$ electron gas trapped in the potential well of galaxy clusters (e.g. Sarazin 1986) has revealed an increasingly complex picture of the intracluster medium (ICM). High resolution X-ray images reveal the presence of small-scale structures of different temperatures (e.g. Donnelly et al. 1999, and references therein). High central densities, lower temperatures, and cooling times significantly shorter than a Hubble time in the centers of many clusters have been interpreted as evidence for cooling flows (e.g. Fabian 1994; White et al. 1997; Allen 2000), where

Send offprint requests to: M. Stickel, e-mail: stickel@mpia-hd.mpg.de

* Based on observations with ISO, an ESA project with instruments funded by ESA Member States (especially the PI countries: France, Germany, The Netherlands and the UK) and with the participation of ISAS and NASA. the cooling gas drops out of thermal equilibrium, possibly leading to the formation of dense clouds and eventually to stars. X-ray spectra and image decomposition indicate an extended excess absorption of soft X-rays with an HI column density of $\approx 10^{21} \mathrm{~cm}^{-2}$ above the galactic foreground (e.g. White et al. 1991; Allen \& Fabian 1997; Sarazin 1997; Allen 2000). However, Arabadjis \& Bregman (2000) find significantly lower or even no additional absorbing column densities in a number of other clusters. A spatially extended EUV emission of non-thermal origin (Enßlin et al. 1999, and references therein) above the expected flux from the hot X-ray gas in at least the Coma and possibly other clusters seems to be fairly established (Lieu et al. 1996a,b; Mittaz et al. 1998; Lieu et al. 1999; Bowyer et al. 1999), although an accompanying low energy X-ray excess emission is uncertain (Dixon et al. 1996; Arabadjis \& Bregman 1999). Overall, these observations indicate a striking departure from a nearly homogeneous 
intracluster gas towards a spatially inhomogeneous multiphase ICM (Bonamente et al. 2001; Lieu et al. 2000).

Despite quite some observational effort, neither the final stage of the cooling intracluster gas nor the material responsible for the excess absorption has been detected at other wavelengths (e.g. Sarazin 1997; Laor 1997; Koekemoer et al. 1998; Miller et al. 1999; Allen 2000, and references therein). It has been suggested that the absorbing material is present as either very cold molecular clouds (Ferland et al. 1994) or dense cold clouds where the shielding allows the formation of dust grains onto which all volatiles have frozen (Daines et al. 1994; Fabian et al. 1994).

The measured line emission of heavy elements (e.g. Mushotzky et al. 1996; Fukazawa et al. 1998) clearly indicates that the ICM contains a processed component, likely the result of galactic winds, stripping of the interstellar medium (ISM) of cluster members by ram pressure, or tidally removed ISM during merging with other galaxy groups. These processes also add dust to the ICM, which in turn is a possible candidate for the excess X-ray absorber. The first direct evidence for dust in the ICM is an observed oxygen $\mathrm{K}$ edge in an X-ray spectrum of the Perseus cluster attributed to neutral material, most likely dust grains condensed out of the ICM (Arnaud \& Mushotzky 1998).

In equilibrium conditions, the predicted intracluster dust (ICD) temperatures are rather uncertain with temperatures in the range 10-20 K (Dwek et al. 1990; Loewenstein \& Fabian 1990; Braine et al. 1995), but possibly much higher (O'Dea et al. 1994; Voit \& Donahue 1995). The thermal emission of the ICD is thus to be expected in the FIR. If not shielded in dense clouds, the high kinetic temperatures destroy dust grains with a typical size of $\approx 0.1 \mu \mathrm{m}$ by sputtering over time scales of a few $10^{8}-10^{9}$ years (Dwek et al. 1990; Tielens et al. 1994; Dwek et al. 1996). Very big $(>10 \mu \mathrm{m})$ dust grains introduced to account for the observed FIR spectra of galaxies (RowanRobinson 1992) would have a significantly longer lifetime of the order a Hubble time. Possibly, the dust destruction is more efficient for smaller $(<0.1 \mu \mathrm{m})$ grains, leaving preferentially behind the bigger grains, which moreover might have a flatter extinction curve than those observed in the Milky Way (Aguirre 1999b). Furthermore, the temperatures in the central cooling flow regions of clusters might generally be lower, which, in addition to the shielding by clouds, would contribute to an increased grain lifetime.

If dust is the absorbing material inferred from the X-ray observations, a significant fraction of the absorbed $\mathrm{X}$-ray radiation of $\gtrsim 10^{43} \mathrm{erg} \mathrm{s}^{-1}$ is re-emitted in the FIR, and dust would then constitute a major coolant of the ICM (Bregman 1992; Sarazin 1997). The FIR emission from ICD would then also be relevant for the correct interpretation of measurements of the Sunyaev-Zeldovich effect in the sub-mm wavelength range (Khersonskii \& Voshchinnikov 1985; Lamarre et al. 1998).

Intracluster dust may reveal itself by optical depth effects in cosmological investigations of distant objects
(Shaver 1987; Masci 1998), a significant fraction of which are viewed through clusters. Historically, this has been the first attempt to find evidence for the existence of ICD (Zwicky 1957, 1962). Despite quite some effort to search for the visual extinction of background quasars and galaxies seen through foreground galaxy clusters, no common consent has been achieved whether properties of background sources are actually affected by the presence of dust in foreground clusters (Girardi et al. 1992; Ferguson 1993; Maoz 1995, and references therein). Aguirre (1999a,b) and Simonsen \& Hannestad (1999) have drawn attention to the possibility that the observed dimming of distant type Ia supernovae might be caused by intervening dust rather than resulting from a non-zero cosmological constant.

From a comparison of Ly $\alpha$ and UV continuum fluxes in 10 cooling flow clusters, $\mathrm{Hu}$ (1992) found an average excess reddening of $E_{B-V} \approx 0.19$ above the galactic foreground absorption. This was attributed to ICD spread out over a large fraction of the cluster volume. Model calculations of the extended FIR emission from ICD were presented by Dwek et al. (1990) and Hu (1992), who concluded that the expected FIR flux was just within reach of the available IRAS data and the then upcoming ISO mission.

The FIR emission as a direct indicator for ICD has been searched for at $60 \mu \mathrm{m}$ and $100 \mu \mathrm{m}$ on IRAS ISSA plates (Wheelock et al. 1994) by Wise et al. (1993). Removing the large scale galactic foreground cirrus emission, evidence for diffuse excess FIR emission of low statistical significance was found in the direction of several out of 56 clusters. Sub-millimeter observations of 11 cooling flow clusters by Annis \& Jewitt (1993) did not detect any emission from dust near the cluster centers. With the more sensitive sub-millimeter bolometer array at the JCMT, Edge et al. (1999) detected the dust emission of the central galaxies from 2 out of 5 cooling flow clusters. However, the latter two studies were insensitive to extended emission of ICD on angular scales larger than a few arc minutes. Hansen et al. (2000) used ISOPHOT FIR maps to search for dust coincindent with the central galaxies of cooling flow clusters, but obtained only inconclusive results.

Compared to FIR maps, strip-scanning measurements crossing the cluster provide a means to search a larger fraction of the cluster volume, and to reach the FIR foreground level far off the cluster centre. Furthermore, the separation of extended intracluster dust emission from the foreground zodiacal and galactic cirrus emission can be accomplished by using observations at two different wavelengths longward of the IRAS bands. This combined technique was employed in a pilot study to search for dust in the intracluster medium of the Coma cluster (Abell 1656, $z=0.0232$ ) with ISOPHOT (Lemke et al. 1996; Lemke \& Klaas 1999), the photometer instrument aboard ISO (Kessler et al. 1996; Kessler 1999). An enhanced $120 \mu \mathrm{m}$ emission within the central region of $\approx 10^{\prime}(\approx 0.3 \mathrm{Mpc}$, $\left.H_{0}=70 \mathrm{~km} \mathrm{~s}^{-1} \mathrm{Mpc}^{-1}, q_{0}=0.5\right)$ diameter of the cluster was observed (Stickel et al. 1998). In a follow-up study, 
Table 1. Observed Abell clusters.

\begin{tabular}{lccccccc}
\hline Cluster & $\alpha_{2000}$ & $\delta_{2000}$ & $\begin{array}{c}l \\
{\left[{ }^{\circ}\right]}\end{array}$ & $\begin{array}{c}b \\
{\left[{ }^{\circ}\right]}\end{array}$ & $\begin{array}{c}\text { Redshift } \\
\text { (1) }\end{array}$ & $\begin{array}{c}\text { Richness- } \\
\text { Class }\end{array}$ & $\begin{array}{c}R_{\text {Abell }} \\
{\left[{ }^{\prime}\right]}\end{array}$ \\
\hline Abell 262 & $(2)$ & $(3)$ & $(4)$ & $(5)$ & $(6)$ & $(7)$ & $(8)$ \\
Abell 400 & $01^{\mathrm{h}} 52^{\mathrm{m}} 46.4^{\mathrm{s}}$ & $+36^{\circ} 09^{\prime} 06^{\prime \prime}$ & 136.58 & -25.09 & 0.0161 & 0 & 110 \\
Abell 496 & $02^{\mathrm{h}} 57^{\mathrm{m}} 38.6^{\mathrm{s}}$ & $+06^{\circ} 02^{\prime} 00^{\prime \prime}$ & 170.24 & -44.94 & 0.0238 & 1 & 75 \\
Abell 1656 (Coma) & $04^{\mathrm{h}} 33^{\mathrm{m}} 37.1^{\mathrm{s}}$ & $-13^{\circ} 15^{\prime} 42^{\prime \prime}$ & 209.57 & -36.48 & 0.0327 & 1 & 56 \\
Abell 2670 & $12^{\mathrm{h}} 59^{\mathrm{m}} 35.7^{\mathrm{s}}$ & $+27^{\circ} 57^{\prime} 38^{\prime \prime}$ & 58.16 & +88.01 & 0.0232 & 2 & 75 \\
Abell 4038 (Klemola 44) & $23^{\mathrm{h}} 54^{\mathrm{m}} 13.7^{\mathrm{s}}$ & $-10^{\circ} 25^{\prime} 09^{\prime \prime}$ & 81.32 & -68.52 & 0.0761 & 3 & 26 \\
\hline
\end{tabular}

Notes:

- Coordinates (Cols. 2, 3) of central sky position of ISO scans.

- Redshifts (Col. 6) and richness classes (Col. 7) taken from NED.

- Abell radii (Col. 8) according to Rudnick \& Owen (1977).

ISOPHOT was used to observe five more galaxy clusters in a similar way, and the results, together with a re-analysis of the Coma data, are described in the following.

\section{Cluster selection}

In the first intracluster dust observation with ISOPHOT (Stickel et al. 1998), the Coma cluster was selected because it is the cluster where early optical extinction studies had found evidence for ICD (Zwicky 1962; Karachentsev \& Lipovetskii 1969), where diffuse optical emission from intracluster material was already known (Welch \& Sastry 1971; Mattila 1977), and where a detailed theoretical study (Dwek et al. 1990) showed that the predicted diffuse FIR emission was within reach for ISOPHOT.

Primary candidates for additional observations were Abell 262 and Abell 2670, since Wise et al. (1993) had found marginal evidence for extended FIR emission on IRAS ISSA plates. Because the Coma cluster is known to undergo merging with smaller galaxy groups (Colless \& Dunn 1996; Vikhlinin et al. 1997; Burns et al. 1994b) the dust inferred from the diffuse FIR emission was interpreted as being transferred rather recently to the ICM by tidal stripping of cluster galaxy ISM (Stickel et al. 1998). Extended FIR emission from the ICM thus might be found preferentially in recent mergers or young clusters, which makes the testable prediction that old clusters should show weak or no extended FIR emission. It was therefore attempted to find more targets along the sequence of X-ray morphologies outlined by Jones \& Forman (1992) and quantified as a possible dynamical and evolutionary sequence by Buote \& Tsai $(1995,1996)$. Particularly, the supposedly young clusters with irregular and the supposedly old virialized clusters with circular symmetric X-ray emission were considered.

Cluster candidates from the list of Buote \& Tsai (1996) were subject to constraints in the sky visibility by ISO and in cluster redshift. The latter was necessary because at too large redshifts only very few non-overlapping sky positions would have been possible to observe with the $3^{\prime}$ wide
ISOPHOT detector inside a characteristic cluster Abell radius, while the nearest clusters would have required prohibitively long integration times to cover a significant fraction of the cluster diameter. Since a re-observation of the Coma cluster became impossible, Abell 4038 was chosen as a closely resembling cluster, particularly with respect to the multipole moments of its X-ray morphology (Buote \& Tsai 1996).

The observed Abell clusters are listed in Table 1, which gives for each cluster (Col. 1) the coordinates, on which the ISOPHOT observations were centered (Cols. 2, 3), its galactic coordinates (Cols. 4, 5), the cluster redshift (Col. 6), the cluster richness class (Col. 7), and the cluster Abell radius according to Rudnick \& Owen (1977) (Col. 8).

\section{Observations}

The observations were carried out with the C200 camera of ISOPHOT (Lemke et al. 1996; Lemke \& Klaas 1999), a $2 \times 2$ pixel array of stressed Ge:Ga with a pixel size of 89". 4 . For each cluster, linear scans across the cluster center at two different position angles were obtained, each of which was observed with both the C_120 and C_180 filters (reference wavelength $120 \mu \mathrm{m}$ and $180 \mu \mathrm{m}$, equivalent widths $47 \mu \mathrm{m}$ and $72 \mu \mathrm{m}$, respectively) at the same sky positions. The positional offset between subsequent sky measurements was $3^{\prime}$, which resulted in almost no detector overlap. The integration time at each sky position was $\approx 50$ s for Abell 1656 and $\approx 80$ s for the other clusters, during which 6-16 integration ramps with 127 non-destructive readouts were obtained.

Details of the observations are listed in Table 2, which gives for each cluster (Col. 1) the date (Col. 2) and ISO revolution number of the observation (Col. 3), the number of sky positions (Col. 4) and the fraction of the Abell radius covered by the scan (Col. 5) at each position angle (Col. 6) together with the total on-target integration time (Col. 7). Finally, Col. 8 gives the zodiacal light contribution for $120 \mu \mathrm{m}$ and $180 \mu \mathrm{m}$ for the observing date in 
Table 2. Observational details.

\begin{tabular}{llcccccc}
\hline Cluster & $\begin{array}{l}\text { Observing } \\
\text { Date }\end{array}$ & $\begin{array}{c}\text { ISO } \\
\text { Revolution }\end{array}$ & \# Steps & $\begin{array}{c}\text { Cluster } \\
\text { Coverage }^{a}\end{array}$ & $\begin{array}{c}\text { Position Angle } \\
{\left[{ }^{\circ}\right]}\end{array}$ & $\begin{array}{c}\text { On-target } \\
\text { Time } \\
{[\mathrm{s}]}\end{array}$ & $\begin{array}{c}\text { Zodiacal Light at } \\
120 \mu \mathrm{m} / 180 \mu \mathrm{m} \\
{[\mathrm{MJy} / \mathrm{sr}]}\end{array}$ \\
\hline \multicolumn{1}{c}{$(1)$} & \multicolumn{1}{c}{$(2)$} & $(3)$ & $(4)$ & $(5)$ & $(6)$ & $(7)$ & $(8)$ \\
\hline Abell 262 & Jan. 7, 1998 & 784 & 31 & 0.82 & 45,135 & 10250 & $2.7 / 1.8$ \\
Abell 400 & Feb. 21, 1998 & 829 & 19 & 0.76 & 40,130 & 6530 & $5.1 / 2.4$ \\
Abell 496 & Mar. 12, 1998 & 848 & 17 & 0.91 & 90 & 2950 & $1.9 / 0.9$ \\
Abell 496 & Mar. 19, 1998 & 855 & 17 & 0.91 & 180 & 2950 & 3480 \\
Abell 1656 (Coma) & Jul. 21, 1996 & 247 & 16 & 0.64 & 36,82 & $2.3 / 1.1$ \\
Abell 2670 & Nov. 21, 1997 & 736 & 17 & 1.96 & 19,84 & 5640 & $3.8 / 1.6$ \\
Abell 4038 (Klemola 44) & Dec. 24, 1997 & 769 & 19 & 0.89 & 120 & 3260 & $3.0 / 1.4$ \\
Abell 4038 (Klemola 44) & Dec. 25, 1997 & 771 & 19 & 0.89 & 30 & 3260 \\
\hline
\end{tabular}

\section{Notes:}

${ }^{a}$ Scan length in units of the Abell radius.

Table 3. Physical properties of observed clusters.

\begin{tabular}{|c|c|c|c|c|c|c|}
\hline Cluster & $\begin{array}{c}\text { X-Ray } \\
\text { Morphology }\end{array}$ & $\begin{array}{r}N_{\mathrm{HI}} \\
{\left[10^{20} \mathrm{~cm}^{-2}\right]}\end{array}$ & $\begin{array}{r}\text { Temperature } \\
{[\mathrm{keV}]}\end{array}$ & $\begin{array}{r}\text { Cooling Flow } \\
{\left[M_{\odot} \mathrm{yr}^{-1}\right]}\end{array}$ & $\begin{array}{r}\text { GasMass } \\
{\left[10^{12} M_{\odot}\right]}\end{array}$ & $\begin{array}{l}L_{\mathrm{XBol}} \\
{\left[10^{44} \mathrm{erg} \mathrm{s}^{-1}\right]}\end{array}$ \\
\hline (1) & $(2)$ & $(3)$ & (4) & (5) & (6) & (7) \\
\hline Abell 262 & elliptical [4] & $\begin{array}{rr}5.3 & {[1]} \\
10.5 & {[4]}\end{array}$ & $\begin{array}{ll}2.4 & {[1]} \\
2.5 & {[2]} \\
1.4 & {[4]}\end{array}$ & $\begin{aligned} 27 & {[3] } \\
9 & {[2] } \\
47 & {[7] }\end{aligned}$ & $\begin{array}{ll}2.5 & {[3]} \\
6.5 & {[2]}\end{array}$ & $\begin{array}{ll}0.9 & {[1]} \\
0.5 & {[2]} \\
0.4 & {[4]}\end{array}$ \\
\hline Abell 400 & irregular $[6,8]$ & $8.8[1]$ & $\begin{array}{ll}2.5 & {[1]} \\
2.2 & {[2]}\end{array}$ & $\begin{array}{c}0[2] \\
0 \quad[10]\end{array}$ & $\begin{array}{ll}11.1 & {[2]} \\
15.0 & {[6]}\end{array}$ & $\begin{array}{ll}0.7 & {[1]} \\
0.6 & {[2]}\end{array}$ \\
\hline Abell 496 & $\begin{array}{c}\text { single } \\
\text { symmetric }[8]\end{array}$ & $\begin{array}{rr}4.4 & {[1]} \\
12.0 & {[5]}\end{array}$ & $\begin{array}{ll}3.9 & {[1]} \\
4.8 & {[2]} \\
3.3 & {[5]}\end{array}$ & $\begin{array}{rr}112 & {[7]} \\
138 & {[2]} \\
95 & {[3]}\end{array}$ & $\begin{array}{rr}2.7 & {[2]} \\
74.1 & {[2]}\end{array}$ & $\begin{array}{ll}5.8 & {[1]} \\
7.9 & {[2]}\end{array}$ \\
\hline $\begin{array}{l}\text { Abell } 1656 \\
\text { (Coma) }\end{array}$ & elliptical [11] & $0.9[1]$ & $\begin{array}{ll}8.3 & {[1]} \\
7.3 & {[2]}\end{array}$ & $\begin{array}{ll}0 & {[3]} \\
0 & {[2]}\end{array}$ & $\begin{array}{ll}27.8 & {[3]} \\
75.6 & {[2]}\end{array}$ & $\begin{array}{rr}12.5 & {[1]} \\
9.1 & {[2]}\end{array}$ \\
\hline Abell 2670 & double $[8,9]$ & $\begin{array}{ll}2.7 & {[1]} \\
3.6 & {[9]}\end{array}$ & $\begin{array}{ll}3.9 & {[1]} \\
3.7 & {[2]} \\
4.2 & {[9]}\end{array}$ & $\begin{array}{rr}41 & {[2]} \\
0 & {[9]} \\
18 & {[12]}\end{array}$ & $40.7 \quad[2]$ & $\begin{array}{ll}3.8 & {[1]} \\
3.5 & {[2]}\end{array}$ \\
\hline $\begin{array}{l}\text { Abell } 4038 \\
\text { (Klemola 44) }\end{array}$ & elliptical [8] & $1.5[1]$ & $3.3[1]$ & $87 \quad[3]$ & $4.3[3]$ & $2.2[1]$ \\
\hline
\end{tabular}

References:

[1] David et al. (1993); [2] White et al. (1997); [3] Peres et al. (1998); [4] David et al. (1996); [5] MacKenzie et al. (1996); [6] Beers et al. (1992); [7] Edge et al. (1992); [8] Buote \& Tsai (1996); [9] Hobbs \& Willmore (1997); [10] Allen et al. (1997); [11] Vikhlinin et al. (1997); [12] Wise et al. (1993)

Col. 2 taken from Kelsall et al. (1998) for all clusters except Abell 2670, which in turn was taken from the yearly averaged zodiacal light map given by Leinert et al. (1998).

Physical properties of the observed clusters are collected in Table 3, which gives for each cluster (Col. 1) the overall X-ray morphology (Col. 2), the galactic foreground column density (Col. 3), the X-ray temperatures (Col. 4), the estimated cooling flow rate (Col. 5), the derived gas mass (Col. 6), and the X-ray luminosity (Col. 7). Where possible, several entries for each quantity together with the literature reference have been listed to give an indication for the spread in the determination of these values. The six observed clusters cover the range from relaxed elliptical (Abell 262) to irregular (Abell 400) X-ray morphologies, from cool (Abell 262, Abell 400) to hot (Abell 1656) X-ray temperatures, from no (Abell 400, Abell 1656) to a significant (Abell 496) cooling flow rate, and about an order of magnitude in galactic $N_{\mathrm{HI}}$ column density. 


\section{Data reduction}

To push the detection of the expected weak signals at these long wavelengths to the limits, and to get confidence in detected features, several different methods for the signal derivation were used in parallel. Already in the case of the Coma cluster (Stickel et al. 1998), the pairwise differences of consecutive ramp read-outs rather than signals from the full ramp fitting have proven to provide the most robust signals, particularly if only a few ramps at each sky position are available. To get rid of pairwise readout differences affected by cosmic ray hits, the robust outlierinsensitive myriad estimator was computed and $15 \%$ of the most deviant signals as measured by the absolute deviation were cut off. This outlier removal is similar to a median absolute deviation trimming, but instead of the initial median, the sample myriad is used to determine the outliers. The sample myriad value in turn is a robust estimator of the mode (most common value) of a distribution but does not require binning of the actual data set, and is easily computed by minimizing a particular cost function with a tuning constant set to a small value (for details see Kalluri \& Arce 1998).

Separate sets of final signals for each sky position were derived from this tail-trimmed pairwise distribution by computing the mean, median, myriad (Kalluri \& Arce 1998), and the annealing M estimator ( $\mathrm{Li}$ 1996). Additionally, the cumulative trimmed distribution was fitted with the analytical function $\left.y=c_{0}\left(1+\exp \left(\left(x-c_{3}\right) / c_{1}\right)\right)^{c_{2}}\right)$, the first derivative of which resembles very closely a Gaussian. In this case, the final signal value was derived from the maximum of the first derivative of the fitting function.

Signals from the much smaller distribution of ramp slopes at each sky position, obtained by fitting first order polynomials to the deglitched readouts of each integration ramp, were derived for comparison. The initial rampfitting step was done within the ISOPHOT Interactive Analysis PIA ${ }^{1}$ version 9.0 (Gabriel et al. 1997). Since the number of ramps for one sky position was rather small, a subsequent efficient rejection of disturbed ramp slopes was not possible. Therefore, only the robust myriad, the annealing $\mathrm{M}$ estimator, and the standard PIA averaging were applied to derive the final signals.

Eventually, the signals derived by all different methods were corrected for signal dependence on ramp integration times to be consistent with calibration observations (Laureijs et al. 2000), dark-current subtracted, and finally flux calibrated with PIA version 9.0/Cal G version 6.0. For the conversion to an absolute flux level, measurements of the ISOPHOT internal Fine Calibration Source (FCS) obtained at the beginning and end of each scan in each filter were used. The signals from the two accompanying

1 The ISOPHOT data presented in this paper were reduced using PIA, which is a joint development by the ESA Astrophysics Division and the ISOPHOT Consortium. The ISOPHOT Consortium is led by the Max-Planck-Institut für Astronomie, Heidelberg.
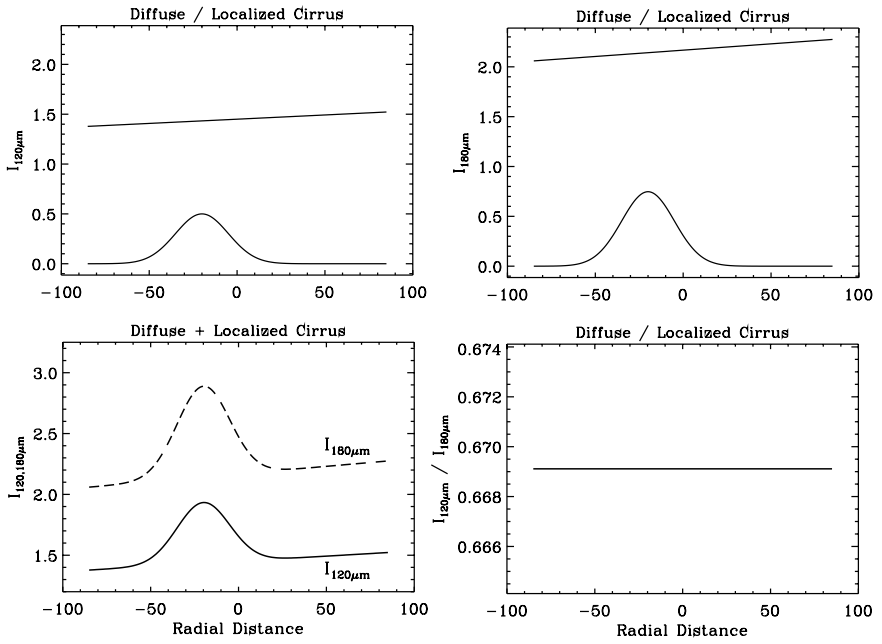

Fig. 1. One-dimensional model of a FIR scan measurement containing cirrus only. A diffuse (straight line) and a localized cirrus component (Gaussian) at $120 \mu \mathrm{m}$ (top left) and $180 \mu \mathrm{m}$ (top right) have similar temperatures, hence the summed intensities at $120 \mu \mathrm{m}$ (bottom left, continuous) and $180 \mu \mathrm{m}$ (bottom left, dashed) have a constant ratio (bottom right).

FCS measurements were linearly interpolated in time to calibrate each sky position of each scan in each filter separately. Because the ISOPHOT calibration has been updated since the publication of the Coma results (Stickel et al. 1998) it was re-analyzed to get a homogeneous set of data.

A comparison of the various signal derivation methods showed that the most robust results as judged by the scatter in the scan signals and $I_{120 \mu \mathrm{m}} / I_{180 \mu \mathrm{m}}$ surface brightness ratios were obtained with the trimmed pairwise distribution using the myriad value as central estimator, while the median and mean values of the trimmed distribution showed a larger scatter. Therefore, only the calibrated data derived from the pairwise myriad method will be considered further. However, the gross features in the $I_{120 \mu \mathrm{m}} / I_{180 \mu \mathrm{m}}$ surface brightness ratios described below were present with decreasing confidence in the $I_{120 \mu \mathrm{m}} / I_{180 \mu \mathrm{m}}$ profile of all different methods used.

\section{Data analysis}

To search for systematic trends in the FIR color profiles across the clusters, the $I_{120 \mu \mathrm{m}} / I_{180} \mu \mathrm{m}$ ratios for each position angle were computed for each detector pixel separately and subsequently averaged. If necessary, at most a single outlier at each sky position was rejected. To further reduce the scatter in the $I_{120 \mu \mathrm{m}} / I_{180 \mu \mathrm{m}}$ ratios, peaks from point sources were removed by linearly interpolating between the two adjacent sky positions. The overall flux ratio distribution was derived by averaging the individual ratios of all pixels and both PAs, applying a global rescaling or outlier rejection if necessary.

Care has to be taken if in addition to the diffuse large scale FIR emission from the galaxy a localized cirrus structure having a similar temperature is crossed. Even the 

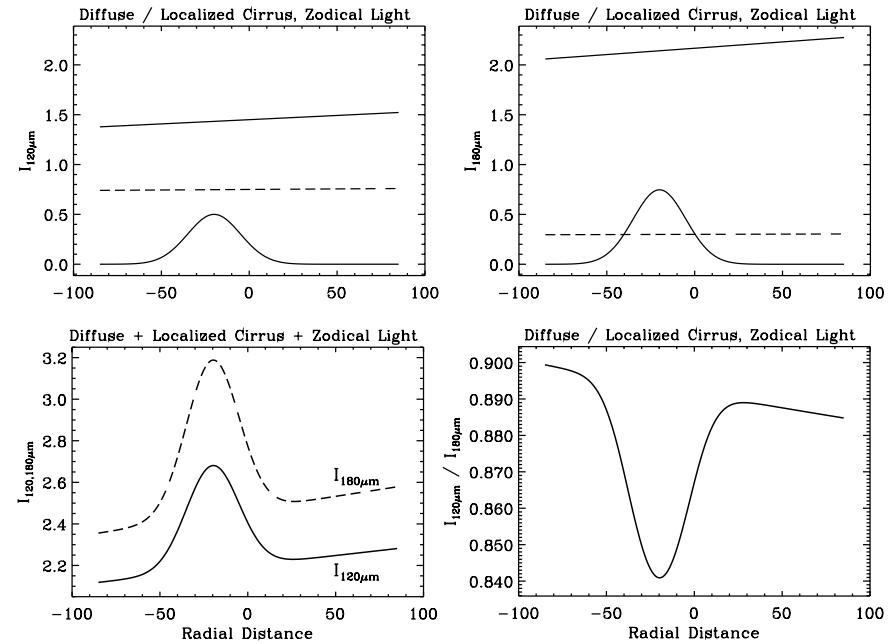

Fig. 2. One-dimensional model of a FIR scan measurement with cirrus and zodiacal light. In addition to the diffuse (continuous straight line) and a localized cirrus component (Gaussian), a zodiacal light component (dashed line) with a much higher temperature is present at $120 \mu \mathrm{m}$ (top left) and $180 \mu \mathrm{m}$ (top right). Although the summed intensities (bottom left) look similar to the case without zodiacal light, their ratio (bottom right) does reflect the profile of the localized cirrus structure as a dip.

small contribution from the zodiacal light, which is weak at FIR wavelengths, will break the degeneracy of the diffuse and localized cirrus component in the $I_{120 \mu \mathrm{m}} / I_{180 \mu \mathrm{m}}$ ratio and mimic an additional extended FIR emitting component.

Specifically, if the $120 \mu \mathrm{m}$ and $180 \mu \mathrm{m}$ surface brightnesses of the diffuse cirrus component are related by $I_{120}^{\mathrm{dif}}=R^{\text {cirr }} \times I_{180}^{\mathrm{dif}}$, and the brightnesses of a localized cirrus structure with a similar temperature are given by $I_{120}^{\text {loc }}=R^{\text {cirr }} \times I_{180}^{\text {loc }}$, the $I_{120 \mu \mathrm{m}} / I_{180 \mu \mathrm{m}}$ ratio $R^{\text {cirr }}$ of galactic cirrus alone is given by

$\frac{I_{120}^{\mathrm{dif}}+I_{120}^{\mathrm{loc}}}{I_{180}^{\mathrm{dif}}+I_{180}^{\mathrm{loc}}}=R^{\mathrm{cirr}}$.

For similar temperatures of both components, this ratio is nearly constant, and almost independent of the brightness profiles of both cirrus components. For an additional zodiacal light component the brightnesses at both wavelengths are related by $I_{120}^{\text {zodi }}=R^{\text {zodi }} \times I_{180}^{\text {zodi }}$, where $R^{\text {zodi }} \neq R^{\text {cirr }}$ because of the different temperatures of galactic cirrus and zodiacal light. by

The total observed $I_{120 \mu \mathrm{m}} / I_{180 \mu \mathrm{m}}$ ratio is then given

$$
\begin{aligned}
\frac{I_{120}^{\text {dif }}+I_{120}^{\text {dif }}+}{I_{180}^{\text {dif }}+I_{180}^{\text {dif }}+} & I_{180}^{\text {zodi }} \\
& +\left(R^{\text {zodi }}-R^{\text {cirr }}\right) \times \frac{I_{180}^{\text {zodi }}}{I_{180}^{\text {dif }}+I_{180}^{\text {dif }}+I_{180}^{\text {zodi }}}
\end{aligned}
$$

Although the brightnesses of the diffuse cirrus component and the zodiacal light vary only slightly across the cluster, the overall ratio is not constant due to the varying brightness of the localized cirrus structure $I_{180 \mu \mathrm{m}}^{\mathrm{dif}}$, and directly reflects the brightness profile of this component.
This behavior can be exemplified by a one-dimensional model, where the diffuse cirrus component $I^{\text {dif }}$ might be approximated by a slanted line with a constant ratio $I_{120}^{\text {dif }} /$ $I_{180}^{\text {dif }}$, while the localized cirrus structure might be modeled for simplicity by a Gaussian with a constant $I_{120}^{\text {dif }} / I_{180}^{\text {dif }}$ ratio equal to that of the diffuse component. The zodiacal light component can also be described by a slanted line to account for a gradient in the zodiacal light distribution along the scan path.

While the model profile of $R^{\text {cirr }}$ (Eq. (1)) is constant (Fig. 1), the zodiacal light component in $R^{\text {cirr+zodi }}$ (Eq. (2)) makes the presence of a localized cirrus component obvious, leading to a characteristic change in the $I_{120 \mu \mathrm{m}} / I_{180 \mu \mathrm{m}}$ ratio along the model scan, which actually reflects the profile of the localized cirrus component (Fig. 2).

On the other hand, if no localized cirrus structure but instead an ICD component described by $I_{120 \mu \mathrm{m}}^{\mathrm{ICD}}=R^{\mathrm{ICD}} \times$ $I_{180 \mu \mathrm{m}}{ }^{\mathrm{IDC}}$ is present with a temperature different from the galactic cirrus, i.e. $R^{\mathrm{ICD}} \neq R^{\mathrm{cirr}}$, then the ratio

$\frac{I_{120}^{\mathrm{dif}}+I_{120}^{\mathrm{ICD}}}{I_{180}^{\mathrm{dif}}+I_{180}^{\mathrm{ICD}}}=R^{\mathrm{cirr}}+\left(R^{\mathrm{ICD}}-R^{\mathrm{cirr}}\right) \times \frac{I_{180}^{\mathrm{ICD}}}{I_{180}^{\mathrm{dif}}+I_{180}^{\mathrm{ICD}}}$

is already varying across the cluster due to the nonconstant ICD component, reflecting the ICD brightness profile. Adding the zodiacal light only changes the level of variation in the observed overall $I_{120 \mu \mathrm{m}} / I_{180 \mu \mathrm{m}}$ ratio, but not the presence of a dip or bump originating in the ICD component.

This behavior provides the possibility to distinguish in the zodiacal light subtracted $I_{120 \mu \mathrm{m}} / I_{180 \mu \mathrm{m}}$ ratio an additional ICD component with a surface brightness varying on scales of a clusters diameter from a localized cirrus component. If a dip or bump is still present in the $I_{120 \mu \mathrm{m}} / I_{180 \mu \mathrm{m}}$ ratio after the subtraction of the zodiacal light component, it can be attributed to an additional FIR component outside the Galaxy and most likely identified with the thermal FIR emission of ICD. On the other hand, a dip or bump which disappears in the $I_{120 \mu \mathrm{m}} / I_{180 \mu \mathrm{m}}$ ratio with the subtraction of the zodiacal light component is most likely due to a localized cirrus structure. Only if the ICD properties (temperature, grain composition, etc.) closely resemble that of galactic cirrus, a characteristic change of the $I_{120 \mu \mathrm{m}} / I_{180 \mu \mathrm{m}}$ ratio from ICD will also disappear with the subtraction of the zodiacal light component, and there is then no possibility to separate the two components with the measurements at two FIR wavelengths.

\section{Results}

\subsection{Abell 262}

Abell 262 is a spiral rich cluster with an E/D type central galaxy (NGC 708) showing dust lanes and diffuse gas with line emission. The cluster X-ray temperature is rather low, and there is independent evidence for a central cooling flow (Table 3). Giovanelli \& Haynes (1985) found that a 

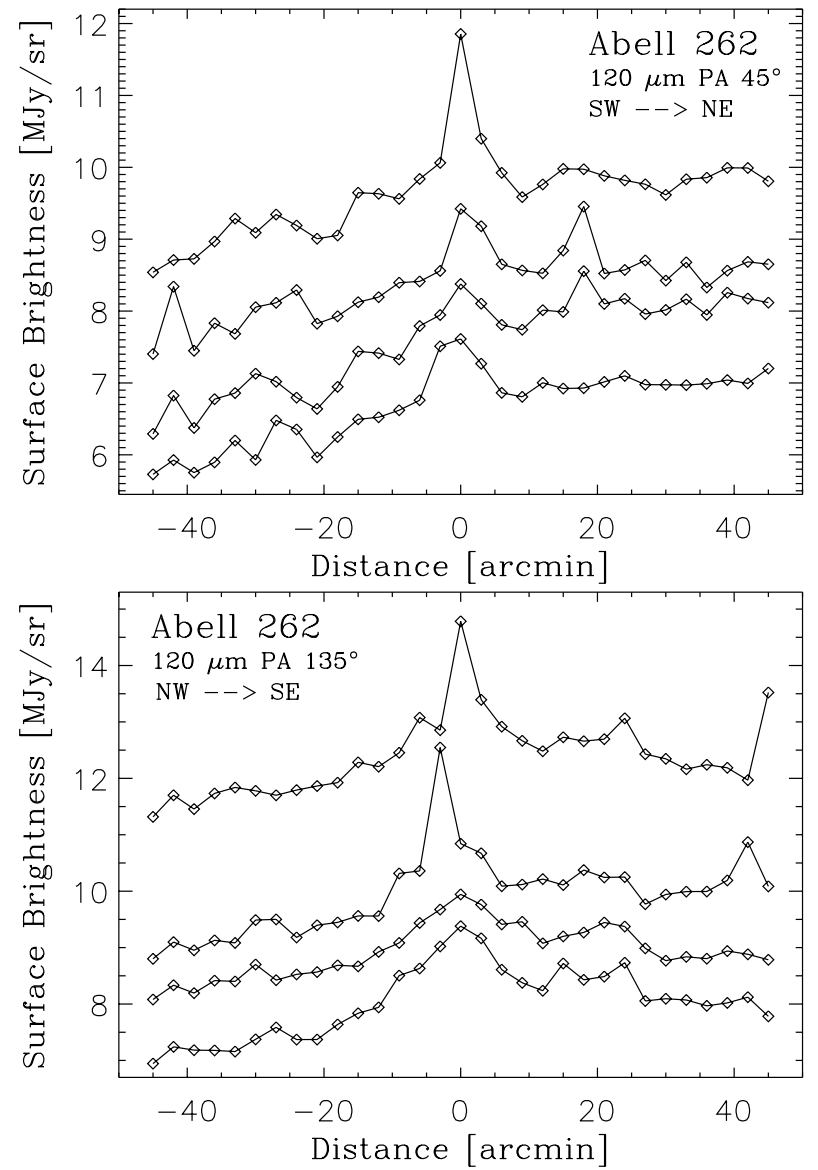

Fig. 3. The observed brightness distributions of the four C200 detector pixels at $120 \mu \mathrm{m}$ for Abell 262 along PA $45^{\circ}$ (top) and PA $135^{\circ}$ (bottom). South lies to the left at negative distances, north to the right. The brightness level is correct only for the lowest data stream. For clarity, the other three pixel data streams are offset arbitrarily. The $180 \mu \mathrm{m}$ brightness distributions (not shown) are quite similar.

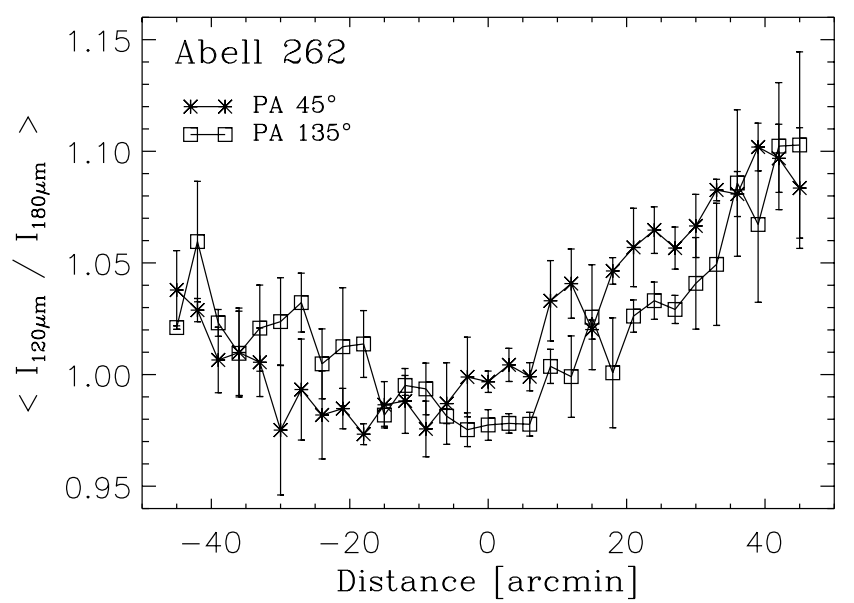

Fig. 4. The raw surface brightness ratios $I_{120 \mu \mathrm{m}} / I_{180 \mu \mathrm{m}}$, averaged over all four detector pixels, along PA $45^{\circ}$ (asterisks) and PA $135^{\circ}$ (squares) as a function of distance from the cluster center of Abell 262. South lies to the left at negative distances, north to the right. Both PAs show a marked south-north asymmetry and a broad depression closely aligned with the cluster center.

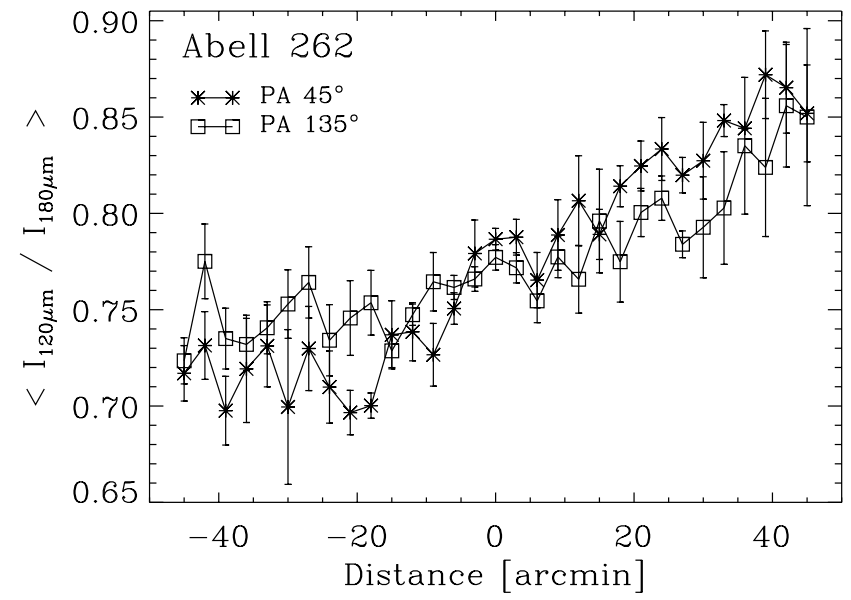

Fig. 5. The surface brightness ratios $I_{120} \mu \mathrm{m} / I_{180 \mu \mathrm{m}}$ after subtraction of the zodiacal light, averaged over all four detector pixels, along PA $45^{\circ}$ (asterisks) and PA $135^{\circ}$ (squares) as a function of distance from the cluster center of Abell 262. South lies to the left at negative distances, north to the right.

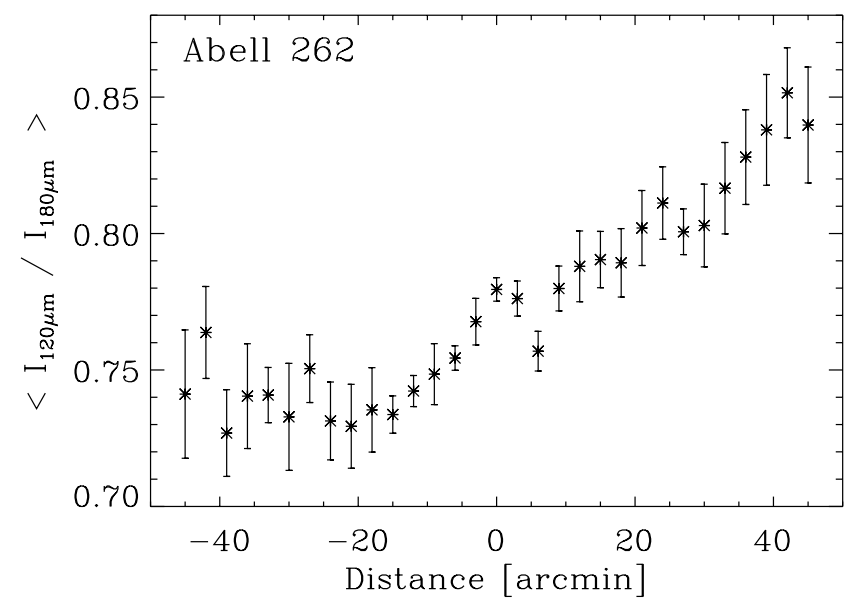

Fig. 6. The overall zodiacal-light subtracted surface brightness ratio $I_{120} \mu \mathrm{m} / I_{180 \mu \mathrm{m}}$ for Abell 262, averaged over both position angles and all detector pixels.

significant fraction of the galaxies inside one Abell radius are deficient in HI, while Bravo-Alfaro et al. (1997) have shown that a number of these galaxies have an asymmetric $\mathrm{HI}$ distribution. Both results are most likely the results of the interaction of the cluster galaxies with the ICM.

The $100 \mu \mathrm{m}$ IRAS HIRES image shows an elongated cloud-like patch of FIR emission, roughly oriented at a PA $\approx 50^{\circ}$, overlaid by filamentary structures. One ISOPHOT scan was executed parallel to this general elongation, while the other one was perpendicular. The extended $100 \mu \mathrm{m}$ emission region lies at the edge of a much larger cirrus structure, and David et al. (1996) argued that the excess X-ray absorber required by the ROSAT PSPC observations is actually galactic cirrus rather than an absorber physically associated with the cluster. The inferred column density of galactic HI is about twice the value usually used for the analysis of X-ray data (Stark et al. 1992), 
indicating significant fluctuations in the galactic HI column density on angular scales $<2^{\circ}$.

However, from emission line ratios $\mathrm{Hu}$ (1992) found evidence for an excess reddening of $E_{B-V} \approx 0.20 \mathrm{mag}$, indicating a cluster internal absorber containing dust. Wise et al. (1993) attempted to subtract the galactic foreground cirrus from IRAS ISSA plates and found evidence for residual diffuse extended emission at $60 \mu \mathrm{m}$ and $100 \mu \mathrm{m}$, which was attributed to intracluster dust, too.

Both ISOPHOT scans show for both position angles the broad bump of extended FIR emission, and confined to one sky position, the presence of an unresolved central point source (Fig. 3). It can not be decided whether this FIR point source is due to the central $\mathrm{cD}$ galaxy, because a spiral galaxy is projected onto its outskirts (Fanti et al. 1982). This confusion was already noted by Bregman et al. (1990). Several other compact sources appear only in the data streams of individual pixels, indicating non-central crossings.

The raw $I_{120 \mu \mathrm{m}} / I_{180} \mu \mathrm{m}$ surface brightness ratios including zodiacal light for both position angles, separately averaged over the four detector pixels, agree remarkably well (Fig. 4), even in the marked north - south asymmetry, and show a very broad minimum somewhat off-center from the nominal cluster centre position. Remarkably, the broad depression completely disappears in the zodiacallight subtracted surface brightness ratios along both PAs (Fig. 5), leaving only an almost linearly increasing overall $I_{120 \mu \mathrm{m}} / I_{180 \mu \mathrm{m}}$ ratio (Fig. 6 ).

\subsection{Abell 400}

A dynamical analysis of Abell 400 was carried out by Beers et al. (1992), who concluded that this spiral rich cluster actually consists of two bound sub-clusters currently undergoing merging. The irregular X-ray morphology (Beers et al. 1992; Buote \& Tsai 1996) and the absence of a cooling flow (White et al. 1997; Allen \& Fabian 1997) also indicates a young dynamical cluster age. The overall $\mathrm{X}$-ray temperature of $\approx 2.5 \mathrm{keV}$ is rather low (David et al. 1993; White et al. 1997).

Although Abell 400 lies at a much higher galactic latitude than e.g. Abell 262, both ISOPHOT scans (Fig. 7) show nevertheless a much higher absolute level of the $120 \mu \mathrm{m}$ surface brightness. The $100 \mu \mathrm{m}$ IRAS HIRES image shows quite a structured region with large patches of cirrus foreground towards Abell 400. Two very bright point sources were detected with one detector pixel only along PA $40^{\circ}$ and $\mathrm{PA} 130^{\circ}$, which have been identified with PGC 11141 (IRAS F02542+0600) and UGC 2444 (IRAS 02558+0606), respectively. Both scans also indicate in the data streams of several pixels the presence of a much weaker compact source confined to the sky position centered on the cluster.

The $I_{120 \mu \mathrm{m}} / I_{180 \mu \mathrm{m}}$ raw surface brightness ratios (Fig. 8) along the two PAs are quite structured on angular scales of $10^{\prime}-20^{\prime}$ without a good overall agreement
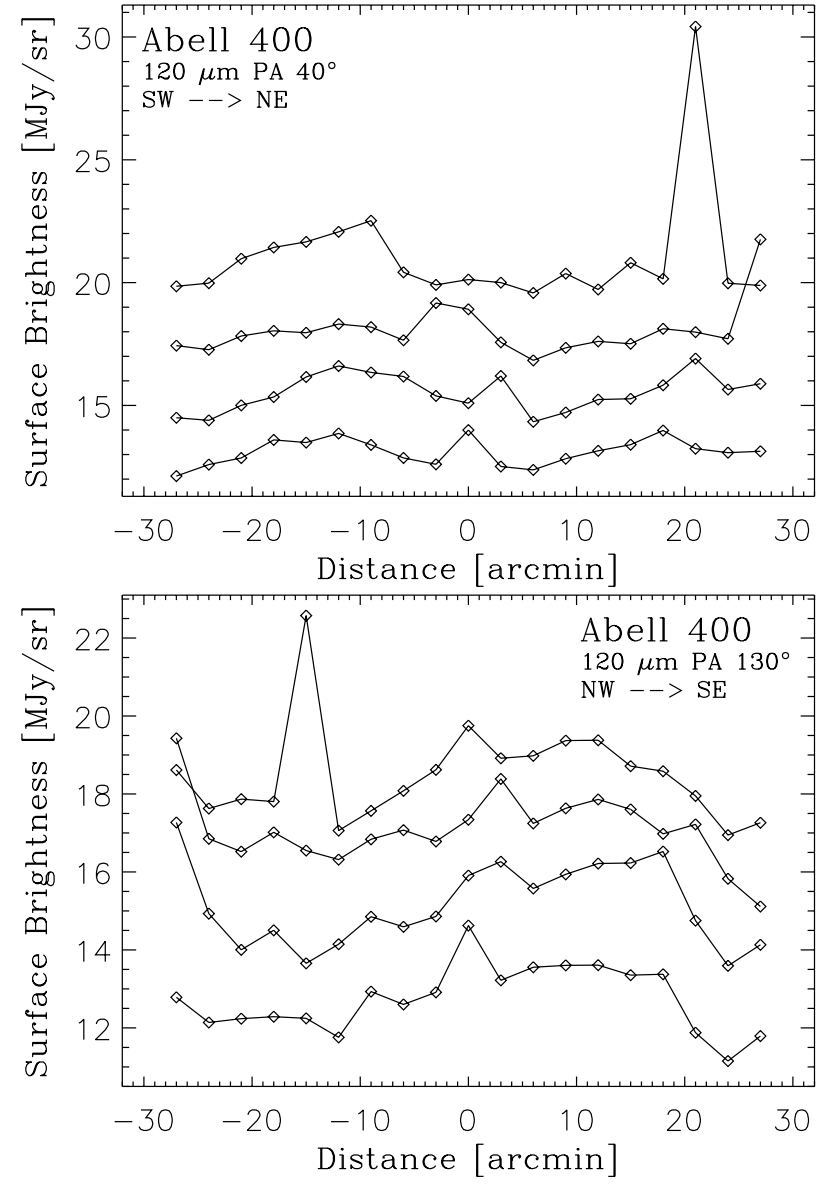

Fig. 7. The observed brightness distributions of the four C200 detector pixels at $120 \mu \mathrm{m}$ for Abell 400 along PA $40^{\circ}$ (top) and PA $130^{\circ}$ (bottom). South lies to the left at negative distances, north to the right. The brightness level is correct only for the lowest data stream. For clarity, the other three pixel data streams are offset arbitrarily. The $180 \mu \mathrm{m}$ brightness distributions (not shown) are quite similar.

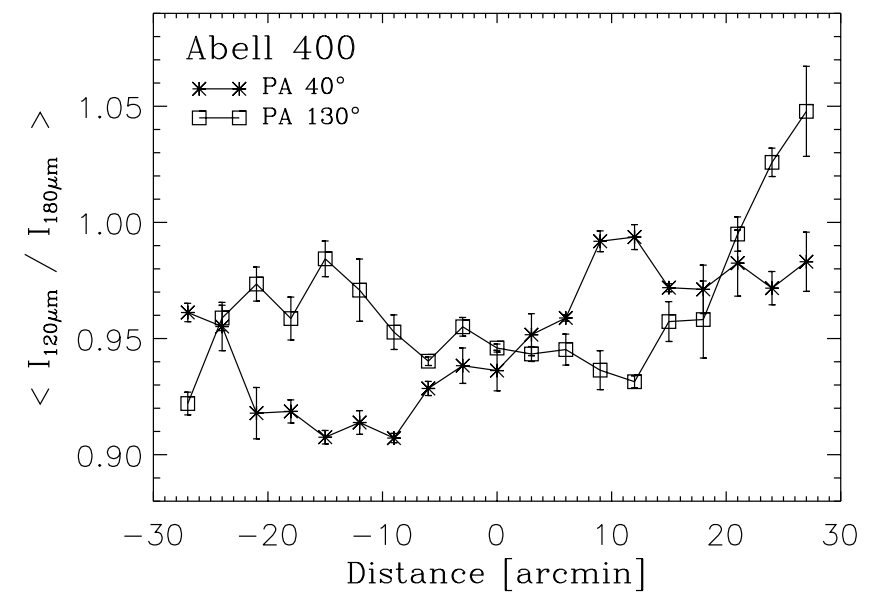

Fig. 8. The raw surface brightness ratios $I_{120 \mu \mathrm{m}} / I_{180 \mu \mathrm{m}}$ averaged over all four detector pixels, along PA $40^{\circ}$ (asterisks) and PA $130^{\circ}$ (squares) as a function of distance from the center of Abell 400. The profiles along the two PAs are rather dissimilar with structures on angular scales of $10^{\prime}-20^{\prime}$. South lies to the left at negative distances, north to the right. 


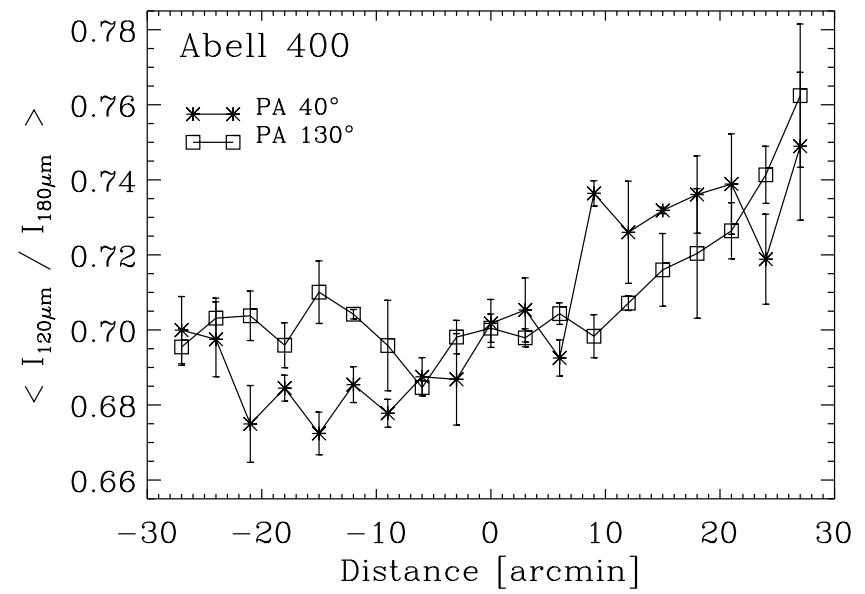

Fig. 9. The surface brightness ratios $I_{120 \mu \mathrm{m}} / I_{180 \mu \mathrm{m}}$ after subtraction of the zodiacal light, averaged over all four detector pixels, along PA $40^{\circ}$ (asterisks) and PA $130^{\circ}$ (squares) as a function of distance from the center of Abell 400. South lies to the left at negative distances, north to the right. The overall shapes of both PAs show a flatter region towards south, and a steepening towards north.

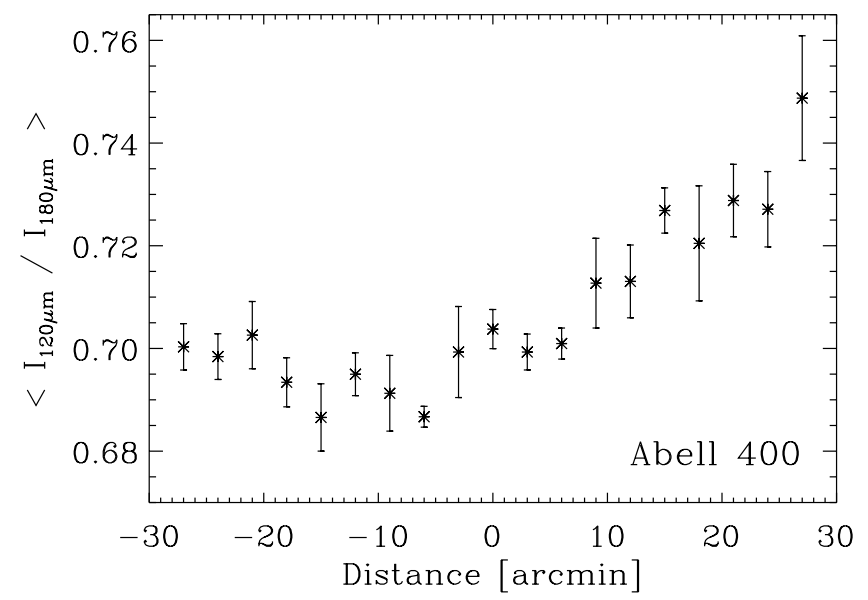

Fig. 10. The overall zodiacal-light subtracted surface brightness ratio $I_{120 \mu \mathrm{m}} / I_{180 \mu \mathrm{m}}$ for Abell 400 , averaged over both position angles and all detector pixels.

of the two profiles. However, the ratio at the scan crossing is in almost perfect agreement, thereby providing an independent check of the flux calibration. After subtraction of the zodiacal light, the $I_{120 \mu \mathrm{m}} / I_{180 \mu \mathrm{m}}$ surface brightness ratios (Fig. 9) of the two PAs are in much better agreement, showing a flatter part towards south and a gradual steepening towards north. This overall behavior is quite easily seen in the overall $I_{120 \mu \mathrm{m}} / I_{180 \mu \mathrm{m}}$ surface brightness ratio (Fig. 10).

\subsection{Abell 496}

The relaxed single symmetric X-ray morphology (Buote \& Tsai 1996), the significant cooling flow (Table 3), and the double-nucleus central $\mathrm{cD}$ galaxy with very small velocity dispersion (Tonry 1985) indicates a dynamically old, relaxed cluster. There is evidence for an X-ray
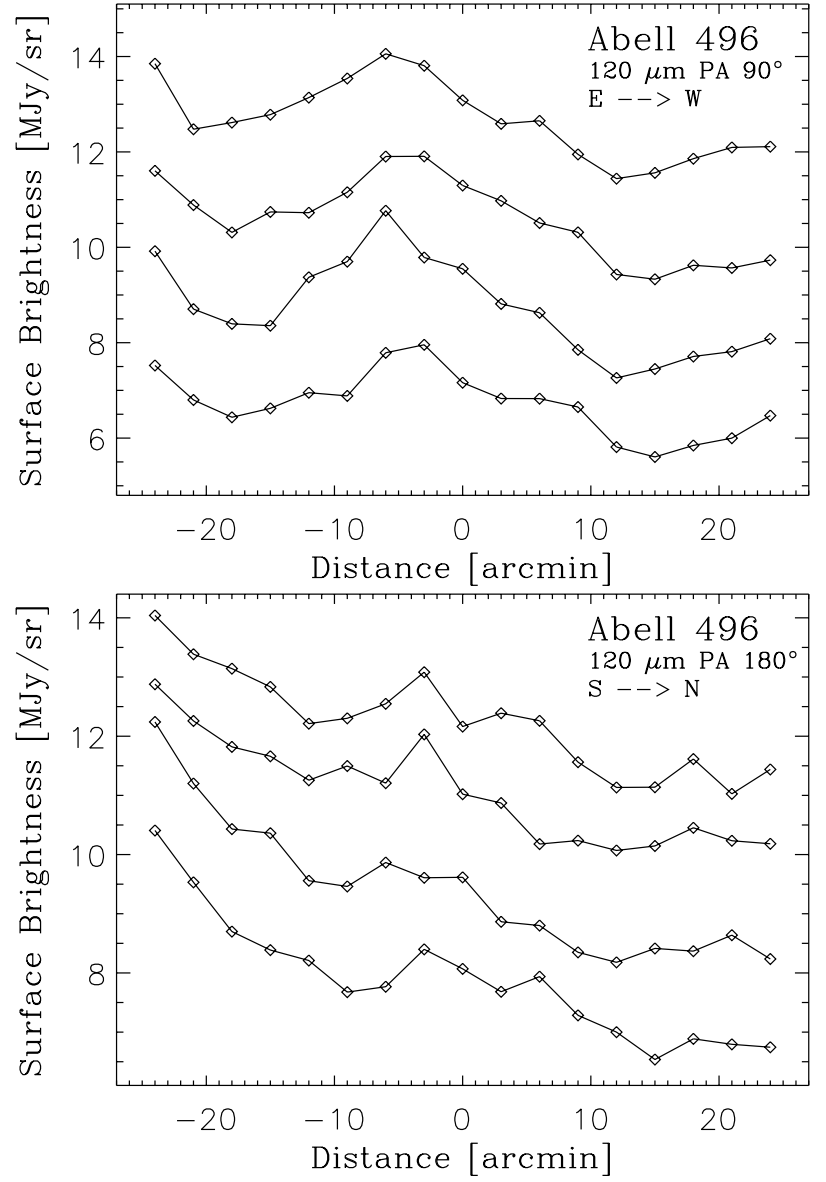

Fig. 11. The observed brightness distributions of the four C200 detector pixels at $120 \mu \mathrm{m}$ for Abell 496 along PA 90 (top) and PA $180^{\circ}$ (bottom). The brightness level is correct only for the lowest data stream. For clarity, the other three pixel data streams are offset arbitrarily. The $180 \mu \mathrm{m}$ brightness distributions (not shown) are quite similar.

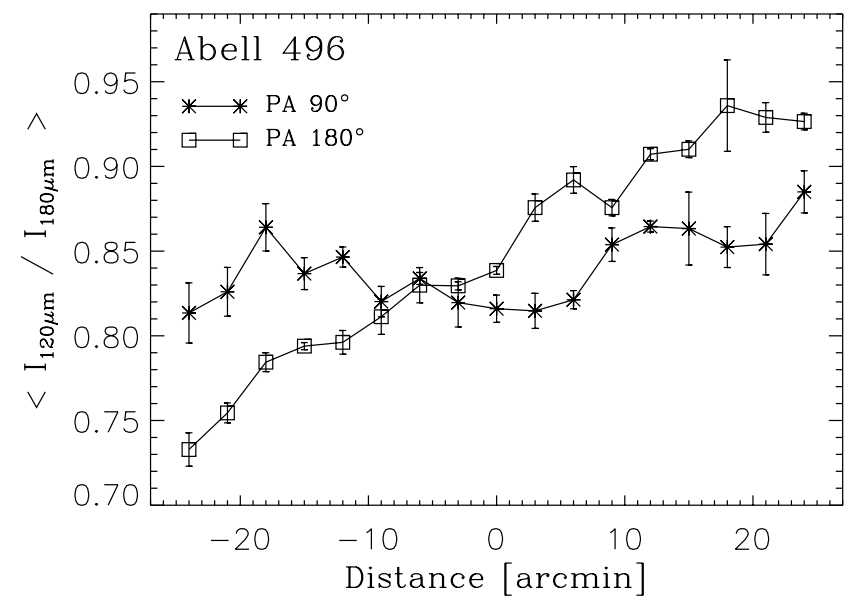

Fig. 12. The raw surface brightness ratios $I_{120 \mu} \mu \mathrm{m} / I_{180 \mu \mathrm{m}}$, averaged over all four detector pixels, along PA $90^{\circ}$ (asterisks, from east towards west) and PA $180^{\circ}$ (squares, from south towards north) as a function of distance from the center of Abell 496. The profile is nearly constant along PA $90^{\circ}$, while PA $180^{\circ}$ shows an almost linear increase. 


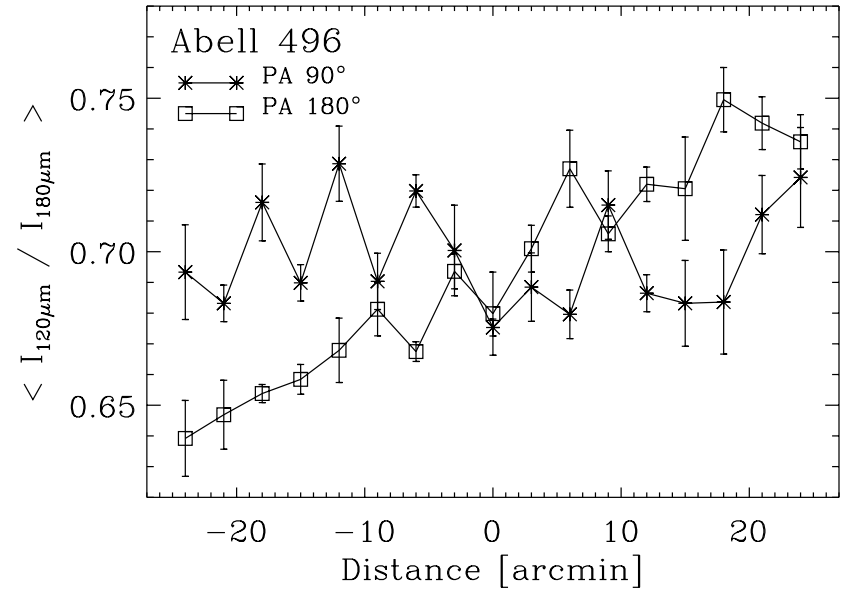

Fig. 13. The surface brightness ratios $I_{120 \mu \mathrm{m}} / I_{180 \mu \mathrm{m}}$ after subtraction of the zodiacal light, averaged over all four detector pixels, along PA $90^{\circ}$ (asterisks, from east towards west) and PA $180^{\circ}$ (squares, from south towards north) as a function of distance from the center of Abell 496.

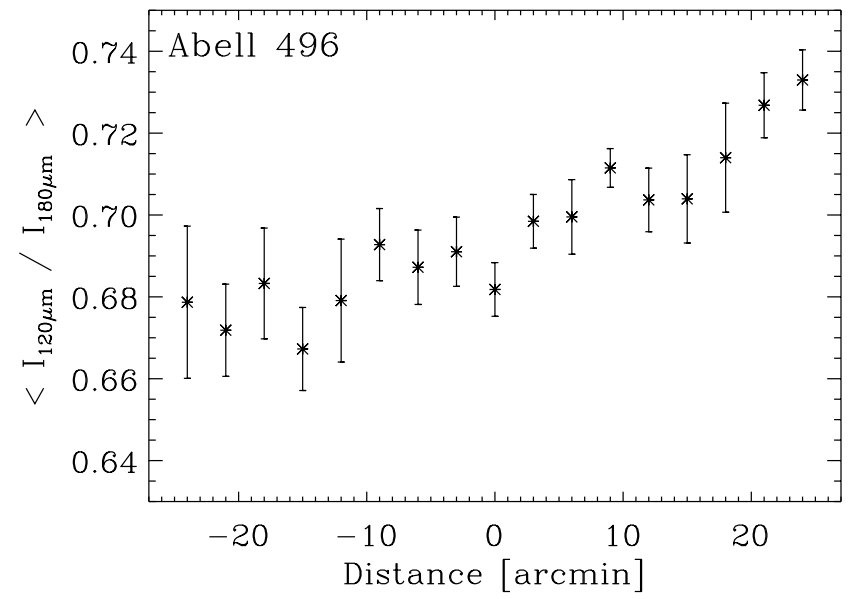

Fig. 14. The overall zodiacal-light subtracted surface brightness ratio $I_{120 \mu \mathrm{m}} / I_{180 \mu \mathrm{m}}$ for Abell 496, averaged over all four detector pixels.

absorption column density above the galactic value across the cluster (White et al. 1994; MacKenzie et al. 1996; Allen et al. 2001), consistent with the excess reddening of $E_{B-V} \approx 0.20$ mag derived from emission line ratios (Hu 1992).

The ISOPHOT scans along both PAs (Fig. 11) are devoid of strong point sources. In the data streams of two pixels along PA $180^{\circ}$, there is an indication for a weak compact source confined to a single sky position. A broad hump offset to the east from the cluster center is visible along PA $90^{\circ}$, and a similar but weaker bump appears to be superposed on the generally decreasing surface brightness profiles along PA $180^{\circ}$.

The two raw $I_{120 \mu \mathrm{m}} / I_{180 \mu \mathrm{m}}$ surface brightness ratios including the zodiacal light (Fig. 12) along PA $180^{\circ}$ and PA $90^{\circ}$ are strikingly different. The former is almost monotonically increasing from south towards north across the cluster, while the latter varies much less across the cluster.
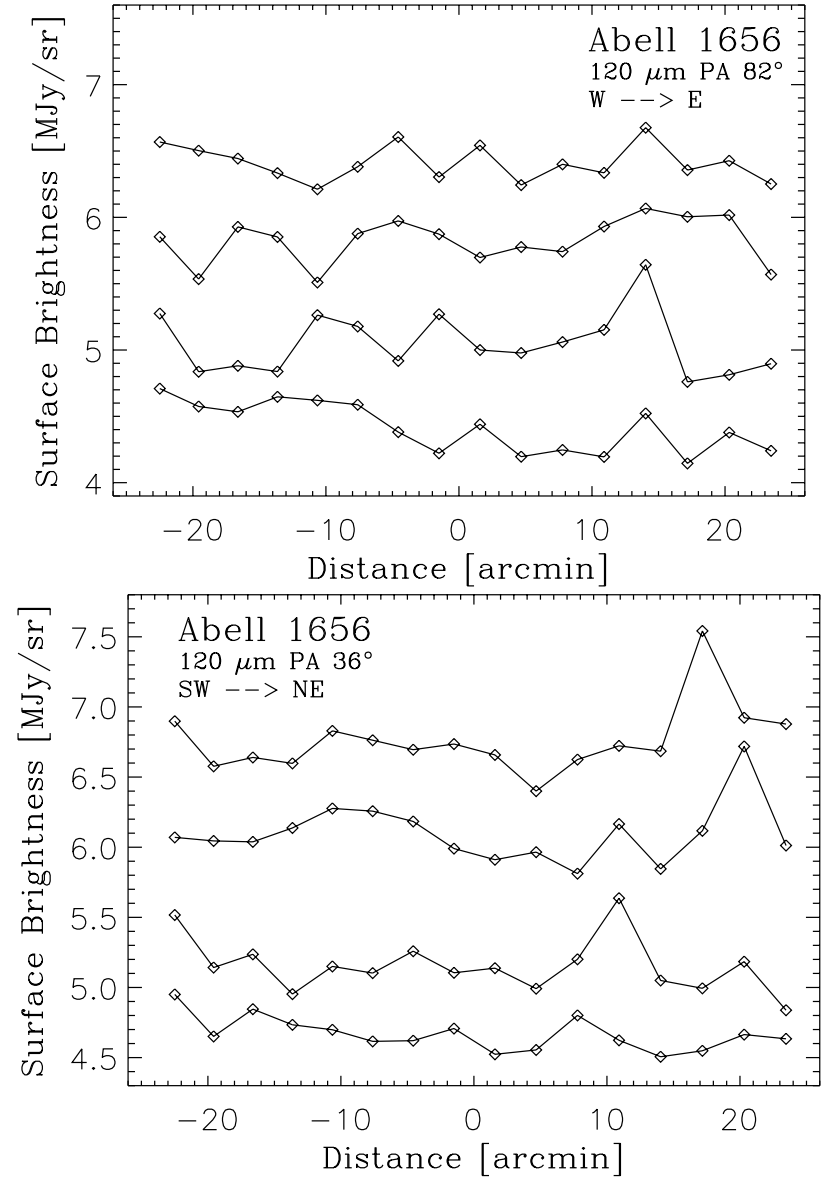

Fig. 15. The observed brightness distributions of the four C200 detector pixels at $120 \mu \mathrm{m}$ for Abell 1656 along PA $82^{\circ}$ (top) and PA $36^{\circ}$ (bottom). The brightness level is correct only for the lowest data stream. For clarity, the other three pixel data streams are offset arbitrarily. The $180 \mu \mathrm{m}$ brightness distributions (not shown) are quite similar.

The color profiles after subtraction of the zodiacal light (Fig. 13) do not change very much, still showing a steady increase from south to north and a rather flat but wiggling behavior along PA $90^{\circ}$. Nevertheless, both profiles were combined to check for the presence of structure on scales of $10^{\prime}-20^{\prime}$. The resulting profile (Fig. 14), however, shows only a gentle rise.

\subsection{Abell 1656 (Coma)}

There is now overwhelming evidence for recent or ongoing merging processes in the Coma cluster from optical and X-ray data (Colless \& Dunn 1996; Vikhlinin et al. 1997; Burns et al. 1994b; Neumann et al. 2001). The early evidence for intracluster light (Welch \& Sastry 1971; Mattila 1977) has recently been confirmed with the detection of additional low surface brightness structures interpreted as tidal debris from recent galaxy interactions (Gregg \& West 1998; Trentham \& Mobasher 1998). With an X-ray temperature of $\approx 8 \mathrm{keV}$, Abell 1656 is the hottest of the 


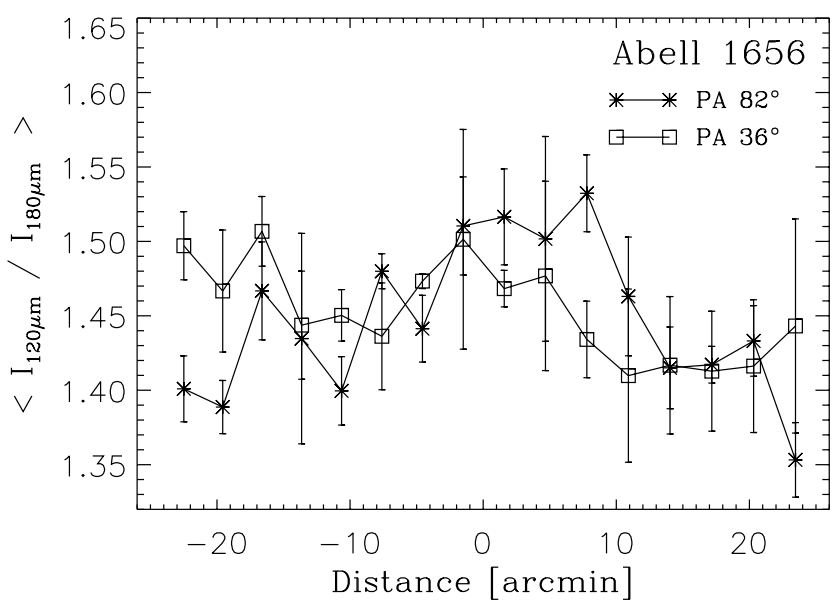

Fig. 16. The raw surface brightness ratios $I_{120 \mu} \mu \mathrm{m} / I_{180 \mu \mathrm{m}}$, averaged over all four detector pixels, along PA $82^{\circ}$ (asterisks) and PA $36^{\circ}$ (squares) as a function of distance from the center of Abell 1656. Both distributions show a broad bump closely aligned with the cluster center, indicating a cold component with $I_{120 \mu \mathrm{m}}>I_{180 \mu \mathrm{m}}$.

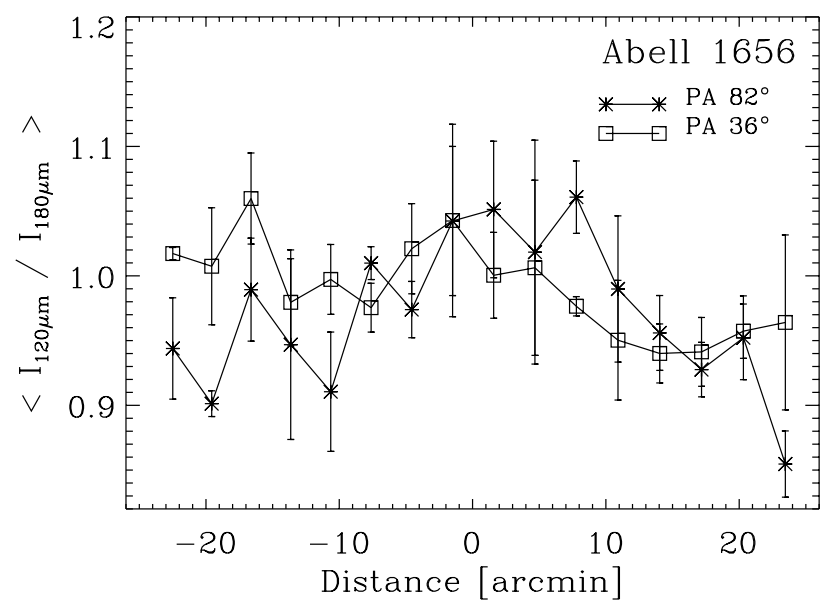

Fig. 17. The surface brightness ratios $I_{120 \mu \mathrm{m}} / I_{180 \mu \mathrm{m}}$ after subtraction of the zodiacal light, averaged over all four detector pixels, along $\mathrm{PA} 82^{\circ}$ (asterisks) and $\mathrm{PA} 36^{\circ}$ (squares) as a function of distance from the center of Abell 1656. The profiles are almost unchanged compared to Fig. 16.

observed clusters, and in fact lies near the upper end of the X-ray luminosity - temperature correlation (Tucker et al. 1998).

Except for a slight decrease in surface brightness level due to the updated ISOPHOT calibration, the scans along $\mathrm{PA} 82^{\circ}$ and $\mathrm{PA} 36^{\circ}$ (Fig. 15) appear almost unchanged compared to those shown in Stickel et al. (1998). However, due to the improved data processing, there is now a much closer agreement between the $I_{120 \mu \mathrm{m}} / I_{180 \mu \mathrm{m}}$ surface brightness ratios along the two PAs (Fig. 16), showing significant deviations only at the outer ends of the scans. An increased $I_{120 \mu \mathrm{m}} / I_{180 \mu \mathrm{m}}$ ratio within $\approx 10^{\prime}$ from the cluster center is apparent, which essentially remains unchanged even after the zodiacal light has been subtracted (Fig. 17). The overall $I_{120 \mu \mathrm{m}} / I_{180 \mu \mathrm{m}}$ ratio averaged over

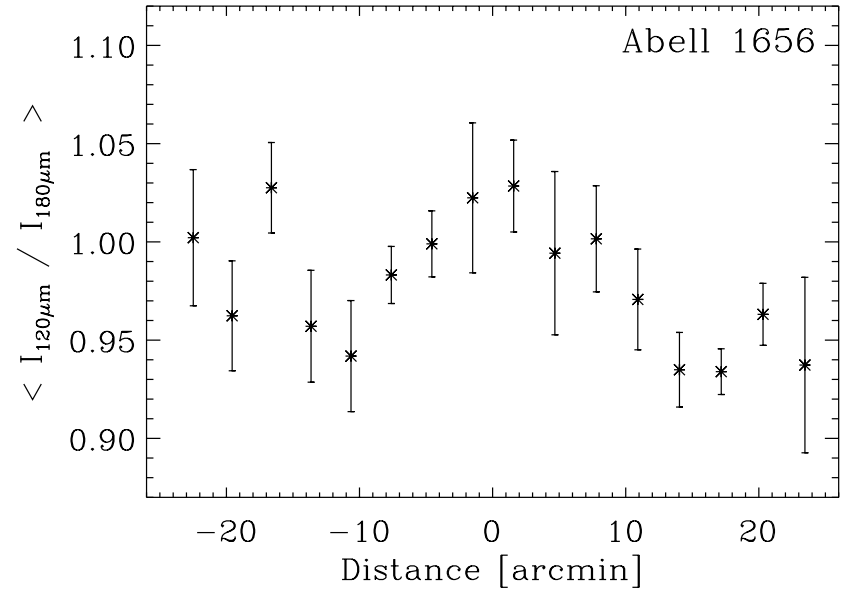

Fig. 18. The overall zodiacal-light subtracted surface brightness ratio $I_{120 \mu \mathrm{m}} / I_{180 \mu \mathrm{m}}$ for Abell 1656 averaged over both position angles and all detector pixels.

both PAs and all detector pixels (Fig. 18) shows the bump quite clearly, which can be quite well represented by a Gaussian with a peak $5 \%$ above a somewhat slanted background.

\subsection{Abell 2670}

The X-ray morphology of Abell 2670 has a double peak in the center (Buote \& Tsai 1996), where the secondary peak is due to several point sources, and a pronounced asymmetry at low flux levels almost along the north-south direction (Hobbs \& Willmore 1997). The ISOPHOT scan position angles were chosen to cover both features.

There are conflicting results regarding the dynamical stage of Abell 2670. Bird (1994), Escalera et al. (1994), and Hobbs \& Willmore (1997) argue in favour of significant substructure as a result of merging, while Sharpless et al. (1988) and Girardi et al. (1995) pointed out the contamination by a foreground cluster. This controversial picture is reinforced by the analysis of X-ray data, where conflicting results about a possible cooling flow have been found (see Table 3).

Extended FIR emission was described by Wise et al. (1993), but judged as uncertain due to the relatively large cluster redshift and small angular extent, and also by Cox et al. (1995). This diffuse emission is easily seen on the $100 \mu \mathrm{m}$ IRAS HiRes image. However, rather than being centered on the cluster, it covers only the western part of the cluster with a north-south elongated patch of low level flux, overlaid with some slightly resolved brighter structures. The central component of these structures is seen in the middle of the overall rather flat surface brightness distributions along both PA $84^{\circ}$ and PA $19^{\circ}$ (Fig. 19).

The $I_{120 \mu \mathrm{m}} / I_{180 \mu \mathrm{m}}$ surface brightness ratios (Fig. 20) from both PAs show a general similarity, a narrow central dip closely aligned with the cluster centre, increasing to approximately the same level at the outer edges of the scans. After subtraction of the zodiacal light, the central dip vanishes in both individual scans (Fig. 21), resulting 

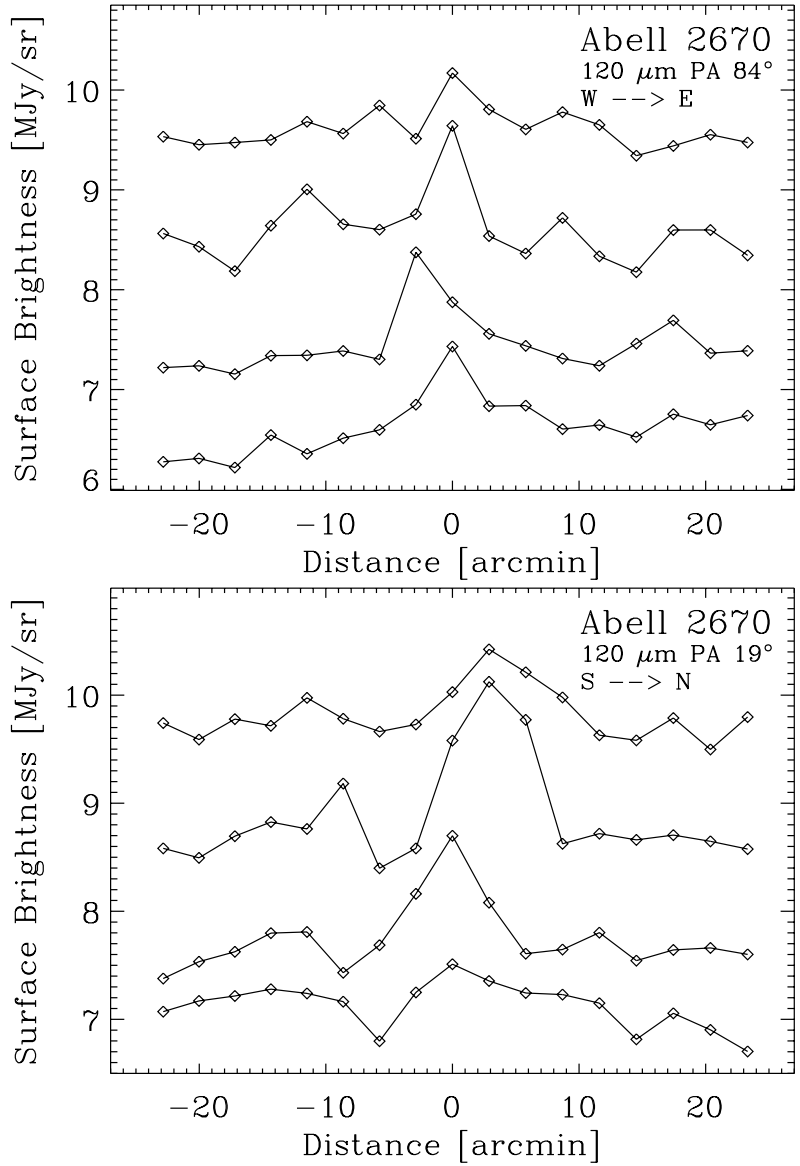

Fig. 19. The observed brightness distributions of the four C200 detector pixels at $120 \mu \mathrm{m}$ for Abell 2670 along PA $84^{\circ}$ (top) and PA $19^{\circ}$ (bottom). The brightness level is correct only for the lowest data stream. For clarity, the other three pixel data streams are offset arbitrarily. The $180 \mu \mathrm{m}$ brightness distributions (not shown) are quite similar.

in an almost flat overall $I_{120 \mu \mathrm{m}} / I_{180 \mu \mathrm{m}}$ ratio across the cluster with only small scale low-level variations (Fig. 22).

\subsection{Abell 4038 (Klemola 44)}

The X-ray morphology of Abell 4038 is similar to that of the Coma cluster, being essentially elliptical with a faint extended source representing a group of galaxies within the cluster (Buote \& Tsai 1996). The X-ray data indicate the presence of a cooling flow (see Table 3 ). The overall galaxy distribution is elongated along $\mathrm{PA} 120^{\circ}$, as is the major axis of the central cD galaxy (Green et al. 1990). One of the ISOPHOT scans was aligned with this direction while the other one was perpendicular.

The overall surface brightness distributions along both PA $120^{\circ}$ and $\mathrm{PA} 30^{\circ}$ (Fig. 23) are rather flat. Only along PA $120^{\circ}$ compact sources show up in the data streams of single detector pixels. The brightest source detected along PA $120^{\circ}$ is actually a background galaxy, identified with IRAS 23440-2816 $(z=0.0495)$.

The $I_{120 \mu \mathrm{m}} / I_{180 \mu \mathrm{m}}$ surface brightness ratios (Fig. 24) along the two PAs agree well, being generally flat with

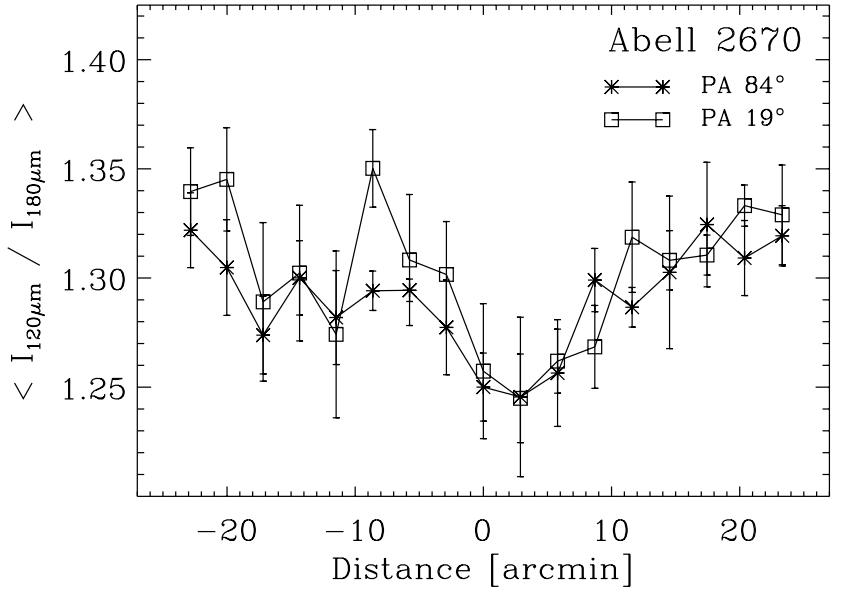

Fig. 20. The raw surface brightness ratios $I_{120 \mu \mathrm{m}} / I_{180 \mu \mathrm{m}}$, averaged over all four detector pixels, along PA $84^{\circ}$ (asterisks) and PA $19^{\circ}$ (squares) as a function of distance from the center of Abell 2670.

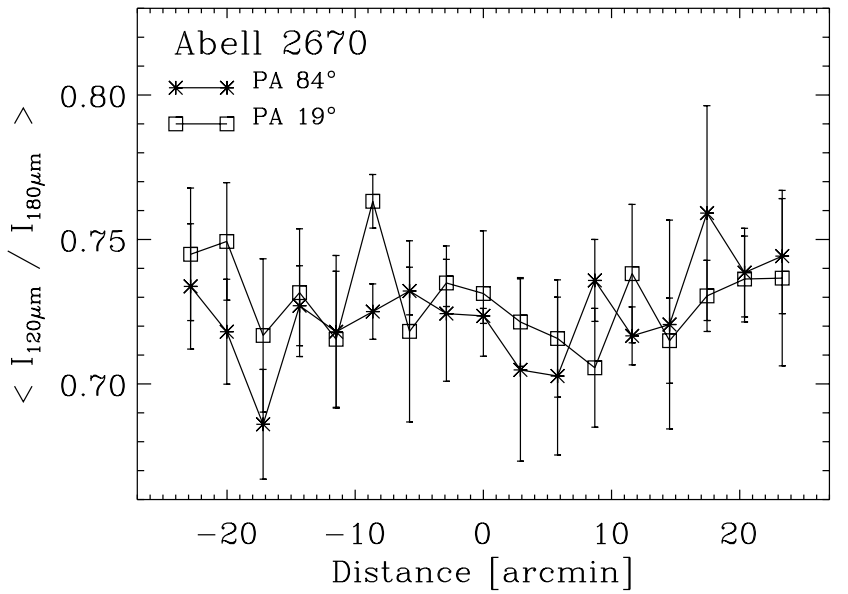

Fig. 21. The surface brightness ratios $I_{120 \mu \mathrm{m}} / I_{180 \mu \mathrm{m}}$ after subtraction of the Zodiacal light, averaged over all four detector pixels, along $\mathrm{PA} 84^{\circ}$ (asterisks) and PA $19^{\circ}$ (squares) as a function of distance from the center of Abell 2670.

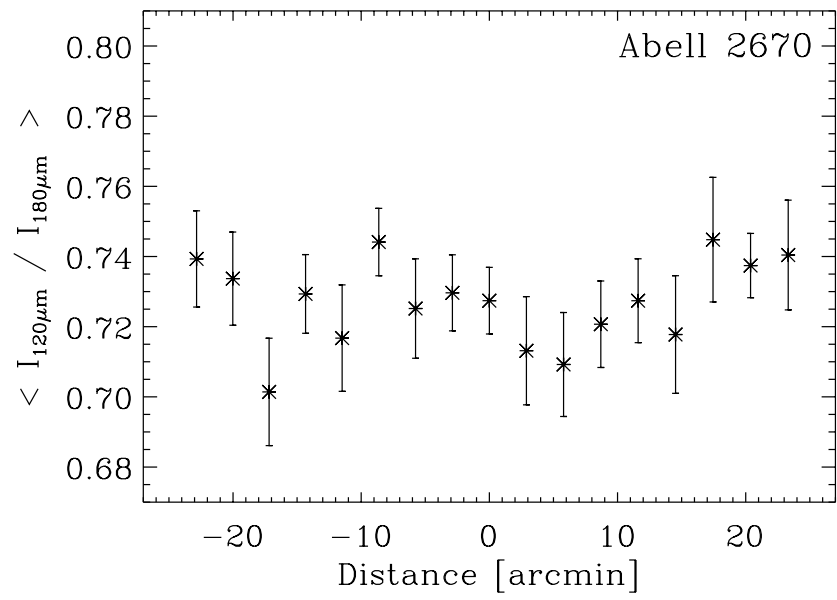

Fig. 22. The overall zodiacal-light subtracted surface brightness ratio $I_{120 \mu \mathrm{m}} / I_{180 \mu \mathrm{m}}$ for Abell 2670 averaged over both position angles and all detector pixels. 

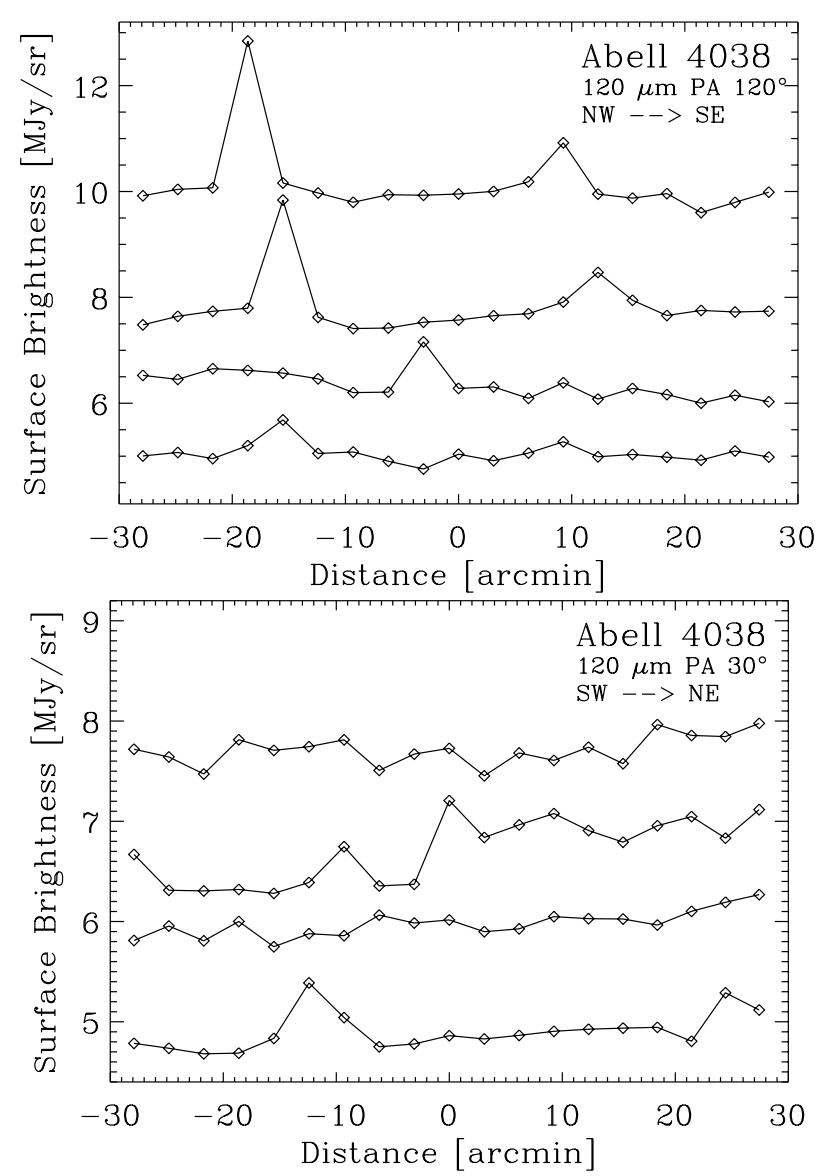

Fig. 23. The observed brightness distributions of the four C200 detector pixels at $120 \mu \mathrm{m}$ for Abell 4038 along PA $120^{\circ}$ (top) and PA $30^{\circ}$ (bottom). The brightness level is correct only for the lowest data stream. For clarity, the other three pixel data streams are offset arbitrarily. The $180 \mu \mathrm{m}$ brightness distributions (not shown) are quite similar. In the scan along PA $30^{\circ}$ (bottom), one pixel data stream shows a marked jump in surface brightness near the cluster center, not seen in any other pixel or wavelength. This is most likely an abrupt change in pixel sensitivity not accounted for by the calibration. This pixel data stream has therefore been ignored in the subsequent analysis.

only small-scale structures. The same behavior is seen after zodiacal-light subtraction (Fig. 25) and eventually in the overall $I_{120 \mu \mathrm{m}} / I_{180 \mu \mathrm{m}}$ profile (Fig. 26).

\section{Discussion}

The striking result of the analysis of the $I_{120} \mu \mathrm{m} / I_{180 \mu \mathrm{m}}$ zodiacal-light subtracted surface brightness ratios is the presence of an easily noticeable bump in only one cluster, namely Abell 1656 (Coma), the cluster with the highest $\mathrm{X}$-ray temperature and no indication of a cooling flow.

Although the raw $I_{120 \mu \mathrm{m}} / I_{180 \mu \mathrm{m}}$ profiles showed structures on scales of $10^{\prime}-20^{\prime}$ also for Abell 262 and Abell 2670, these structures disappeared with the subtraction of the zodiacal light component. This indicates that an additional FIR emitting component outside the Galaxy is present in the direction of Abell 1656 while galactic

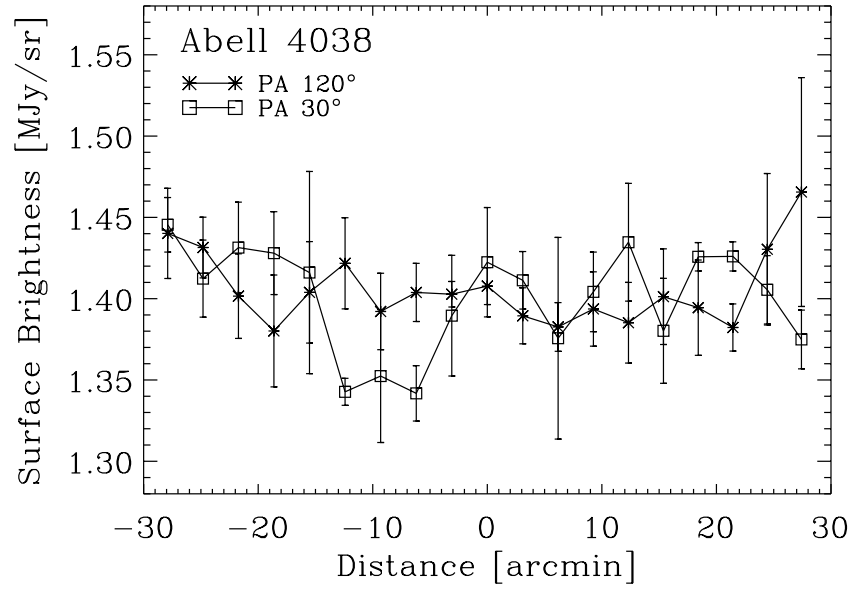

Fig. 24. The raw surface brightness ratios $I_{120 \mu} \mu \mathrm{m} / I_{180 \mu \mathrm{m}}$, averaged over all four detector pixels, along PA $120^{\circ}$ (asterisks, average of four pixels) and PA $30^{\circ}$ (squares, average of three pixels) as a function of distance from the center of Abell 4038.

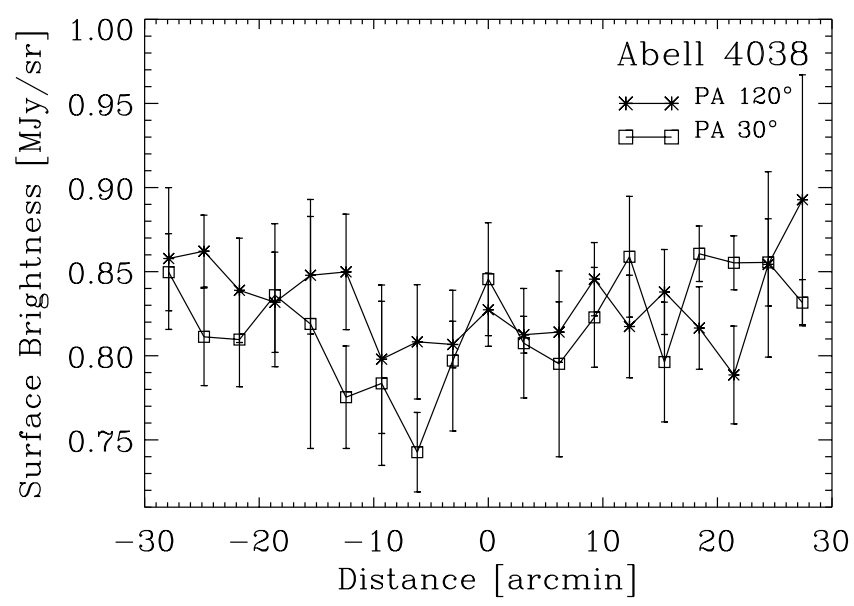

Fig. 25. The surface brightness ratios $I_{120 \mu \mathrm{m}} / I_{180 \mu \mathrm{m}}$ after subtraction of the zodiacal light, averaged over all four detector pixels, along PA $120^{\circ}$ (asterisks, average of four pixels) and PA $30^{\circ}$ (squares, average of three pixels) as a function of distance from the center of Abell 4038.

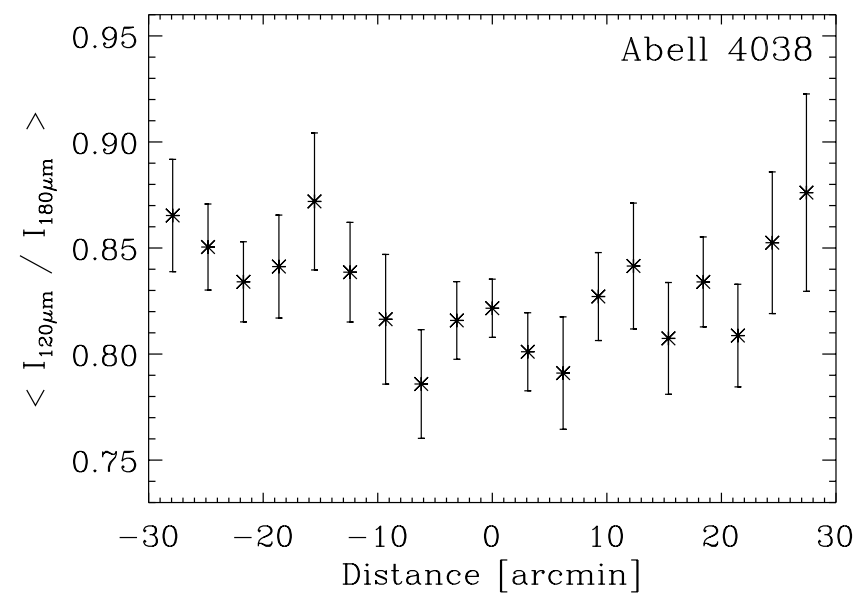

Fig. 26. The overall zodiacal-light subtracted surface brightness ratio $I_{120 \mu \mathrm{m}} / I_{180 \mu \mathrm{m}}$ for Abell 4038 averaged over both position angles and all detector pixels. 
cirrus foreground emission close to the line-of-sight is responsible for the structures in the color profiles of Abell 262 and Abell 2670. This is in clear contrast to the findings of Wise et al. (1993), who ascribed residual FIR emission towards Abell 262 and Abell 2670 found on IRAS ISSA plates to ICD. However, in these two as well as the other three observed clusters, the presence of ICD cannot be excluded completely, because it might be present at very low levels or have properties similar to galactic cirrus. In the former case, the signature is easily lost in the noise, while in the latter case there is no way of discriminating it against the foreground galactic dust even with photometric measurements at additional FIR wavelengths.

The bump in the $I_{120 \mu \mathrm{m}} / I_{180 \mu \mathrm{m}}$ ratio in Abell 1656 (Coma) might be caused by the integrated FIR emission of the unresolved cluster galaxies, if their ISM had different temperatures and/or dust properties than the foreground galactic cirrus (Quillen et al. 1999). Unless the galaxy content of Abell 1656 were rather special, the other clusters should also show a characteristic signature in their $I_{120 \mu \mathrm{m}} / I_{180 \mu \mathrm{m}}$ profiles, which, however, is not observed. Moreover, the FIR color temperature determination of a large sample of normal galaxies selected from the ISOPHOT Serendipity Survey (Stickel et al. 2000) showed the ubiquitous presence of dust in the ISM with temperatures of $T \approx 17 \mathrm{~K}$ similar to the Galaxy.

In principle, the Sunyaev-Zeldovich effect might be another cluster intrinsic cause for extended excess emission at FIR and sub-mm wavelengths. However, even for very hot clusters, the effect at the peak wavelength of $\approx 800 \mu \mathrm{m}$ is small (e.g. Komatsu et al. 1999), and vanishes shortward of $\approx 300 \mu \mathrm{m}$ (Chase et al. 1987; Rephaeli 1995), making it very unlikely as a cause for the observed systematic changes in the $I_{120 \mu \mathrm{m}} / I_{180 \mu \mathrm{m}}$ profiles.

The most likely interpretation of the observed spatially extended bump in the $I_{120 \mu \mathrm{m}} / I_{180 \mu \mathrm{m}}$ ratio of Abell 1656 is therefore the presence of intracluster dust mixed with the hot X-ray emitting electron gas. The excess surface brightness indicates that the intracluster dust has properties different from the foreground galactic cirrus such as temperature, grain sizes, grain composition, or emissivity, otherwise no signature in the $I_{120 \mu \mathrm{m}} / I_{180 \mu \mathrm{m}}$ ratios would be discernible.

The observed overall zodiacal light subtracted $I_{120 \mu \mathrm{m}} / I_{180 \mu \mathrm{m}}$ ratio $R^{\mathrm{obs}}$ can be written as

$R^{\mathrm{obs}}=\frac{I_{120}^{\mathrm{cirr}}+I_{120}^{\mathrm{ICD}}}{I_{180}^{\mathrm{cirr}}+I_{180}^{\mathrm{ICD}}}$

where $I_{120}^{\text {cirr }}$ and $I_{120}^{\mathrm{ICD}}$ are the surface brightnesses of the foreground galactic cirrus and the excess emission of the cluster at $120 \mu \mathrm{m}$, respectively, and $I_{180}^{\mathrm{cirr}}$ and $I_{180}^{\mathrm{ICD}}$ the corresponding brightnesses at $180 \mu \mathrm{m}$. To quantify the bump in the overall zodiacal-light subtracted $I_{120 \mu \mathrm{m}} / I_{180 \mu \mathrm{m}}$ surface brightness ratio of Abell 1656, it was modelled as the sum of a Gaussian, presumed to result from the cluster dust emission $I^{\mathrm{ICD}}$, and a first order polynomial from the large scale cirrus foreground $I^{\text {cirr }}$. The cirrus foreground can then be interpolated at the position of the cluster centre, giving the central $I_{120}^{\text {cirr }} / I_{180}^{\text {cirr }}$ ratio

$R^{\mathrm{cirr}}=\frac{I_{120}^{\mathrm{cirr}}}{I_{180}^{\text {cirr }}}$

of the cirrus alone.

For the case of $R^{\text {obs }}>R^{\text {cirr }}$, the two relations (Eqs. (4), (5)) can be re-arranged to give

$I_{120}^{\mathrm{ICD}}=I_{180}^{\mathrm{cirr}} \times\left[\left(R^{\mathrm{obs}}-R^{\mathrm{cirr}}\right)+R^{\mathrm{obs}} \frac{I_{180}^{\mathrm{ICD}}}{I_{180}^{\mathrm{cirr}}}\right]$.

Under the assumption that the cluster dust emission is weak compared to the cirrus foreground, i.e. $I_{180}^{\mathrm{ICD}} \ll I_{180}^{\mathrm{cirr}}$, it can further be rearranged to

$I_{120}^{\mathrm{ICD}} \simeq\left(I_{180}^{\mathrm{cirr}}+I_{180}^{\mathrm{ICD}}\right) \times\left(R^{\mathrm{obs}}-R^{\mathrm{cirr}}\right)$.

This gives the cluster excess surface brightness $I_{120}^{\mathrm{ICD}}$ in terms of the observed overall $I_{120 \mu \mathrm{m}} / I_{180 \mu \mathrm{m}}$ ratio $R^{\mathrm{obs}}$, the interpolated $I_{120 \mu \mathrm{m}} / I_{180 \mu \mathrm{m}}$ ratio $R^{\text {cirr }}$ of the cirrus, and the observed total surface brightness at $180 \mu \mathrm{m}$.

For Abell $1656, R^{\text {obs }}-R^{\text {cirr }}=0.07$, with a characteristic e-folding angular scale of $\sigma_{\mathrm{ICD}} \approx 5^{\prime}$, and $I_{180}^{\mathrm{cirr}}+I_{180}^{\mathrm{ICD}}=$ $3.1 \mathrm{MJy} / \mathrm{sr}$, resulting in a cluster excess peak surface brightness $I_{120}^{\mathrm{ICD}} \approx 0.2 \mathrm{MJy} / \mathrm{sr}$. Assuming that the fitted one-dimensional Gaussian is representative of an underlying circular symmetric two-dimensional excess flux distribution, the integrated (total) excess fluxes computed from $F_{\text {ICD }}=2 \pi \sigma_{\text {ICD }}^{2} I^{\text {ICD }}$, the volume of the two-dimensional Gaussian with a peak height equal to the derived excess surface brightness and a width equal to the fitted onedimensional Gaussian, is $F_{\mathrm{ICD}} \approx 2.8 \mathrm{Jy}$. This corresponds to a monochromatic FIR luminosity $L_{\mathrm{ICD}}=4 \pi D^{2} \nu F_{\mathrm{ICD}}$ of $L_{\mathrm{ICD}} \approx 8.6 \times 10^{43} \mathrm{erg} / \mathrm{s}$, where $D$ is the cluster luminosity distance.

The absence of a clear sign of cluster excess emission in the individual scans of Abell 1656 at both wavelengths gives strict upper limits for the ICD excess surface brightness of $I_{120}^{\mathrm{ICD}}<0.4 \mathrm{MJy} / \mathrm{sr}$. Under the assumption that the profile of the ICD excess emission at both wavelengths can be approximated by a Gaussian and the cirrus foreground surface brightness varies linearly across the cluster, this absolute upper limit can be transformed to a lower limit of for the peak surface brightness ratio $I_{120}^{\mathrm{ICD}} / I_{180}^{\mathrm{ICD}}$ of the excess emission. This is done by fitting the ratio of the presumed surface brightness profiles (for each wavelength a Gaussian added to a slanted background) to the overall $I_{120 \mu \mathrm{m}} / I_{180 \mu \mathrm{m}}$ ratio, subject to the constraint not to exceed the upper limit, yielding $I_{120}^{\mathrm{ICD}} / I_{180}^{\mathrm{ICD}} \gtrsim 2$. This lower limit in the $I_{120}^{\mathrm{ICD}} / I_{180}^{\mathrm{ICD}}$ ratio transforms into a lower limit in the dust color temperature of $T \gtrsim 30 \mathrm{~K}$, assuming a blackbody (Planck) function modified by a frequency dependent emissivity $F_{\nu}=\nu^{\beta} B_{\nu}\left(T_{\mathrm{D}}\right)$ with exponent $\beta=2$.

An estimation of the intracluster dust mass can be derived by using

$M_{\mathrm{D}}=D^{2} F_{\nu}\left[\kappa_{\lambda} B_{\nu}\left(T_{\mathrm{D}}\right)\right]^{-1}$ 
(Hildebrand 1983; Draine 1990), where $F_{\nu}$ is the total excess flux density at $120 \mu \mathrm{m}, T_{\mathrm{D}}$ the dust color temperature, and $\kappa_{\lambda}$ the dust opacity. With a dust opacity of $3 \mathrm{~m}^{2} / \mathrm{kg}$, which lies in the middle of the range of currently considered values (Draine 1990), a dust temperature of $T_{\mathrm{D}} \approx 30 \mathrm{~K}$ and the excess flux of $F_{\mathrm{ICD}}=2.8 \mathrm{Jy} \mathrm{a}$ dust mass of $M_{\mathrm{D}} \approx 10^{7} M_{\odot}$ is derived. This dust mass is only exemplary, since it depends strongly on the assumed dust color temperature and the dust opacity.

It should be noted that a dust opacity, which already included the conversion to gas mass with a constant galactic gas-to-dust ratio, had been used in the first publication of the Coma observations (Stickel et al. 1998). The newly derived dust mass for Abell 1656 is therefore considerably lower than that given before. Consequently, the gas-to-dust ratio using a gas mass of $M_{\text {gas }} \approx 10^{13} M_{\odot}$ is extremely high $\left(\approx 10^{6}\right)$. Furthermore, the low inferred dust mass leads under standard assumptions about the dust properties (Whittek 1992) to a negligible amount of visual extinction $\left(A_{V} \ll 0.1 \mathrm{mag}\right)$, in contrast to the reported optical extinction of $\approx 0.3 \mathrm{mag}$ (Zwicky 1962; Karachentsev \& Lipovetskii 1969), and in accord with the analysis of the IRAS data by Dwek et al. (1990).

\section{Conclusions}

Spatially extended FIR excess emission from dust distributed in the intracluster medium has been searched for in a small sample of six Abell clusters covering a wide variety in cluster properties, such as X-ray morphology and temperature, cluster age, richness, and distance, by using ISOPHOT scan measurements at $120 \mu \mathrm{m}$ and $180 \mu \mathrm{m}$ wavelength. After subtraction of the zodiacal light, the characteristic signature of a dip or bump on angular scales of $10^{\prime}-20^{\prime}$ is only seen in Abell 1656 (Coma), a hot, dynamically young cluster with optical and X-ray signs of interactions with smaller galaxy groups.

The evidence from IRAS ISSA plates for FIR emission from intracluster dust in Abell 262 and Abell 2670 (Wise et al. 1993) could not be confirmed. Although the raw (including zodiacal light) $I_{120 \mu \mathrm{m}} / I_{180} \mu \mathrm{m}$ profiles did show a dip, it disappeared after the zodiacal light had been subtracted. This behavior is indicative of a localized patch of galactic foreground cirrus, which happens to lie along the line of sight to these clusters. This strikingly underlines the necessity of interpreting FIR measurements, even flux ratios at several wavelengths, carefully by taking into consideration the ever-present cirrus structures in conjunction with the zodiacal light, which can produce a quite counterintuitive behavior.

This result does seem to support the simple picture put forward in Stickel et al. (1998), that only young clusters currently undergoing merging show the signature of intracluster dust, which might indicate that intracluster dust is primarily brought into the cluster from the outside, either by merging with external galaxy groups or possibly by steady infall of intercluster material (Popescu et al. 2000).
The FIR observations of the six clusters, particularly those with a strong cooling flow inferred from X-ray observations, do not seem to support the picture of cold clouds dropping out of the cooling flow and shielding intracluster dust from sputtering, or even dust originating in the dense centers of cooling flow clusters. The rather low inferred dust mass in Abell 1656 and the non-detection of the characteristic signature of ICD in the other five clusters can be taken as evidence that the X-ray excess absorption seen in cooling flow clusters is likely not due to the presence of intracluster dust. Particularly, no excess X-ray absorption is required for Abell 1656 (Allen et al. 2001), the only cluster where evidence for extended FIR emission has been found in this study. The absence of a signature for intracluster dust in the clusters with strong indication for cooling flows is in agreement with several other unsuccessful searches for the final stages of the cooled material, and adds additional doubt on the correctness of material condensations associated with the cooling flow model.

Finally, intracluster dust appears not be responsible for the the observed dimming of the high - redshift supernovae, and the contribution to the FIR extragalactic background light is likely also vanishingly small.

Acknowledgements. The development and operation of ISOPHOT were supported by MPIA and funds from Deutsches Zentrum für Luft- und Raumfahrt (DLR, formerly DARA). The ISOPHOT Data Centre at MPIA is supported by Deutsches Zentrum für Luft- und Raumfahrt (DLR) with funds of Bundesministerium für Bildung und Forschung, grant, No. 50 QI98013. The authors are responsible for the content of this publication.

This research has made use of the Digitized Sky Survey, produced at the Space Telescope Science Institute, NASA's Astrophysics Data System Abstract Service, the Simbad Database, operated at CDS, Strasbourg, France, and the NASA/IPAC Extragalactic Database (NED) which is operated by the Jet Propulsion Laboratory, California Institute of Technology, under contract with the National Aeronautics and Space Administration.

We thank Eli Dwek and Rick Arendt for useful comments on a draft of the manuscript.

\section{References}

Aguirre, A. N. 1999a, ApJ, 512, L9

Aguirre, A. N. 1999b, ApJ, 525, 583

Allen, S. W. 2000, MNRAS, 315, 269

Allen, S. W., \& Fabian, A. C. 1997, MNRAS, 286, 583

Allen, S. W., Fabian, A. C., Johnstone, R. M., Arnaud, K. A., \& Nulsen, P. E. J. 2001, MNRAS, 322, 589

Annis, J., \& Jewitt, D. 1993, MNRAS, 264, 593

Arnaud, K. A., \& Mushotzky, R. F. 1998, ApJ, 501, 119

Arabadjis, J. S., \& Bregman, J. N. 1999, ApJ, 514, 607

Arabadjis, J. S., \& Bregman, J. N. 2000, ApJ, 536, 144

Beers, T. C., Gebhardt, K., Huchra, J. P., et al. 1992, ApJ, 400, 410

Bird, C. 1994, ApJ, 422, 480

Bonamente, M., Lieu, R., \& Mittaz, J. P. D. 2001, ApJ, 546, 805 
Bowyer, S., Berghöfer, T., \& Korpela, E. J. 1999, ApJ, 526, 592

Braine, J., Wyrowski, E., Radford, S. J. E., Henkel, C., \& Lesch, H. 1995, A\&A, 293, 315

Bravo-Alfaro, H., Szomoru, A., Cayatte, V., Balkowski, C., \& Sancisi, R. 1997, A\&A, 126, 537

Bregman, J. N., McNamara, B. R., \& O'Connell, R. W. 1990, ApJ, 351, 406

Bregman, J. N. 1992, in Clusters and Superclusters of Galaxies, ed. A. C. Fabian, NATO ASI, 366, 49

Buote, D. A., \& Tsai, J. C. 1995, ApJ, 452, 522

Buote, D. A., \& Tsai, J. C. 1996, ApJ, 458, 27

Burns, J. O., Roettiger, K., Ledlow, M., \& Klypin, A. 1994b, ApJ, 427, L87

Chase, S. T., Joseph, R. D., Robertson, N. A., \& Ade, P. A. R. 1987, MNRAS, 225, 171

Colless, M., \& Dunn, A. M. 1996, ApJ, 458, 435

Cox, C. V., Bregman, J. N., \& Schombert, J. M. 1995, ApJS, 99, 405

Daines, S. J., Fabian, A., \& Thomas, P. 1994, MNRAS, 268, 1060

David, L. P., Slyz, A., Jones, C., et al. 1993, ApJ, 412, 479

David, L. P., Jones, C., \& Forman, W. 1996, ApJ, 473, 692

Dixon, W. V. D., Hurwitz, M., \& Ferguson, H. C. 1996, ApJ, 469, L77

Donnelly, R. H., Markevitch, M., Forman, W., et al. 1999, ApJ, 513, 690

Draine, B. T. 1990, in The Interstellar Medium in Galaxies, ed. H. A. Thronson Jr., \& J. M. Schull (Dordrecht: Kluwer), 483

Dwek, E., Rephaeli, Y., \& Mather, J. 1990, ApJ, 350, 104

Dwek, E., Foster, S. M., \& Vancura, O. 1996, ApJ, 457, 244

Edge, A. C., Stewart, G. C., \& Fabian, A. C. 1992, MNRAS, 258,177

Edge, A. C., Ivison, R. J., Smail, I., Blain, A. W., \& Kneib, J.-P. 1999, MNRAS, 306, 599

Enßlin, T. A., Lieu, R., \& Biermann, P. L. 1999, A\&A, 344, 409

Escalera, E., Biviano, A., Girardi, M., et al. 1994, ApJ, 423, 539

Fabian, A. C. 1994, ARA\&A, 32, 277

Fabian, A. C., Johnstone, R., \& Daines, S. 1994, MNRAS, 271, 737

Fanti, C., Fanti, R., Feretti, L., et al. 1982, A\&A, 105, 200

Ferguson, H. C. 1993, MNRAS, 263, 343

Ferland, G., Fabian, A., \& Johnstone, R. 1994, MNRAS, 266, 399

Fukazawa, Y., Makishima, K., Tamura, T., et al. 1998, PASJ, 50, 187

Gabriel, C., Acosta-Pulido, J., Heinrichsen, I., et al. 1997, Astronomical Data Analysis Software and Systems VI, ed. G. Hunt, \& H. E. Payne, ASP Conf. Ser., 125, 108

Giovanelli, R., \& Haynes, M. P. 1985, ApJ, 292, 404

Girardi, M., Mezzetti, M., Giuricin, G., \& Mardirossian, F. 1992, ApJ, 394, 442

Girardi, M., Biviano, A., Giuricin, G., Mardirossian, F., \& Mezzetti, M. 1995, ApJ, 438, 527

Green, M. R., Gowin, J. G., \& Peach, J. V. 1990, MNRAS, 243,159

Gregg, M. D., \& West, M. J. 1998, Nature, 396, 549

Hansen, L., Jørgensen, H. E., Nørgaard-Nielsen, H. U., et al. 2000, A\&A, 362, 133

Hildebrand, R. H. 1983, QJRAS, 24, 267
Hu, E. M. 1988, in Cooling Flows in Clusters and Galaxies, ed. A. C. Fabian (Kluwer, Dordrecht), 73

Hu, E. M. 1992, ApJ, 391, 608

Hobbs, I. S., \& Willmore, A. P. 1997, MNRAS, 289, 685

Jones, C., \& Forman, W. 1992, in Clusters and Superclusters of Galaxies, ed. A. C. Fabian, NATO ASI, 366, 49

Kalluri, S., \& Arce, G. R. 1998, IEEE Trans. Sig. Proc., 46, 322

Karachentsev, I. D., \& Lipovetskii, V. A. 1969, Soviet Phys., 12, 909

Kessler, M. F., Steinz, J. A., \& Anderegg, M. E., et al. 1996, A\&A, 315, L27

Kessler, M. F. 1999, in The Universe as seen by ISO, ESA-SP 427, ed. P. Cox, \& M. F. Kessler, 23

Kelsall, T., Weiland, J. L., Franz, B. A., et al. 1998, ApJ, 508, 44

Khersonskii, V., \& Voshchinnikov, N. 1985, Astrophys., 20, 605

Koekemoer, A. M., O'Dea, C. P., Baum, S. A., et al. 1998, ApJ, 508, 608

Komatsu, E., Kitayama, T., Suto, Y., et al. 1999, ApJ, 516, L1

Lamarre, J. M., Giard, M., Pointecouteau, E., et al. 1998, ApJ, 507, L5

Laor, A. 1997, ApJ, 483, L103

Laureijs, R. J., Klaas, U., Richards, P. J., Schulz, B., \& Ábrahám, P. 2000, The ISO Handbook, vol. V: PHT - The Imaging Photo-Polarimeter, version 1.1, SAI/99-069/dc

Lemke, D., Abolins, J., Abraham, P., et al. 1996, A\&A, 315, L64

Lemke, D., \& Klaas, U. 1999, in The Universe as seen by ISO, ESA-SP 427, ed. P. Cox, \& M. F. Kessler, 55

Leinert, Ch., Bowyer, S., Haikala, L., et al. 1998, A\&AS, 127, 1

Li, S. Z. 1996, Pattern Recog., 29, 159

Lieu, R., Mittaz, J. P. D., Bowyer, S., et al. 1996a, Science, 274,1335

Lieu, R., Mittaz, J. P. D., Bowyer, S., et al. 1996b, ApJ, 458, L5

Lieu, R., Ip, W.-H., Axford, W. I., \& Bonamente, M. 1999, ApJ, 510, L25

Lieu, R., Bonamente, M., \& Mittaz, J. P. D. 2000, A\&A, 364 497

Loewenstein, M., \& Fabian, A. C. 1990, MNRAS, 242, 120

MacKenzie, M., Schlegel, E., \& Mushotzky, R. 1996, ApJ, 468, 86

Masci, F. J. 1998, Publ. Astron. Soc. Austr., 15, 299

Mattila, K. 1977, A\&A, 60, 425

Maoz, D. 1995, ApJ, 455, L115

Miller, E. D., Bregman, J. N., \& Knezek, P. M. 1999 [astro-ph/9911021]

Mittaz, J. P. D., Lieu, R., \& Lockman, F. J. 1998, ApJ, 498, L17

Mushotzky, R., Loewenstein, M., Arnaud, K. A., et al. 1996, ApJ, 466, 686

Neumann, D. M., Arnaud, M., \& Gastaud, R. 2001, A\&A, 365, L74

O'Dea, C. P., Baum, S. A., Maloney, P. R., Tacconi, L. J., \& Sparks, W. B. 1994, ApJ, 422, 467

Peres, C., Fabian, A., Edge, A., et al. 1998, MNRAS, 298, 416

Popescu, C., Tuffs, R., Fischera, J., \& Völk, H. 2000, A\&A, 354,480

Quillen, A. C., Rieke, G. H., Rieke, M. J., Caldwell, N., \& Engelbracht, C. 1999, ApJ, 518, 632

Rephaeli, Y. 1995, ApJ, 445, 33 
Rowan-Robinson, M. 1992, MNRAS, 258, 787

Rudnick, L., \& Owen, F. N. 1977, AJ 82, 1

Sarazin, C. L. 1986, Rev. Mod. Phys., 58, 1

Sarazin, C. L. 1997, in Galactic and Cluster Cooling Flows, ed. N. Soker, ASP Conf. Ser., 115, 172

Sharpless, R. M., Ellis, R. S., \& Gray, P. M. 1988, MNRAS, 231, 479

Shaver, P. A. 1987, in High Redshift and Primeval Galaxies, ed. J. Bergeron, D. Kunth, B. Rocca-Volmerange, \& J. Tran Than Van (Gif-sur-Yvette: Éditions Frontières), 429

Simonsen, J. T., \& Hannestad, S. 1999, A\&A, 351, 1

Stark, A. A., Gammie, C., Wilson, R., et al. 1992, ApJS, 79, 77

Stickel, M., Lemke, D., Mattila, K., Haikala, L. K., \& Haas, M. 1998, A\&A 329, 55

Stickel, M., Lemke, D., Klaas, U., et al. 2000, A\&A, 359, 865

Tielens, A. G. G. M., McKee, C. F., Seab, C. G., \& Hollenbach, D. J. 1994, ApJ, 431, 321

Tonry, J. L. 1985, AJ, 90, 2431

Trentham, N., \& Mobasher, B. 1998, MNRAS, 293, 53
Tucker, W., Blanco, P., Rappoport, S., et al. 1998, ApJ, 496, L5

Vikhlinin, A., Forman, W., \& Jones, C. 1997, ApJ, 474, L7

Voit, G. M., \& Donahue, M. 1995, ApJ, 452, 164

Welch, G. A., \& Sastry, G. N. 1971, ApJ, 169, L3

Wheelock, S. L., Gautier, T. N., \& Chillemi, J. 1994, JPL Publication, 94-11

White, D. A., Fabian, A. C., Johnstone, R. M., Mushotzky, R. F., \& Arnaud, K. A. 1991, MNRAS, 252, 72

White, D. A., Jones, C., \& Forman, W. 1997, MNRAS, 292, 419

White, R. E., Day, C. S. R., Hatsukade, I., \& Hughes, J. P. 1994, ApJ, 433, 583

Whittek, D. C. B. 1992, Dust in the Galactic Environment (Institute of Physics Publishing, Bristol)

Wise, M. W., O'Connell, R. W., Bregman, J. N., \& Roberts, M. S. 1993, ApJ, 405, 94

Zwicky, F. 1957, Morphological Astronomy (Springer, Berlin)

Zwicky, F. 1962, in Problems in Extragalactic Research, ed. G. C. McVittie (New York: Macmillan), 149 